\title{
Matching of heavy-light flavour currents between HQET at order $1 / m$ and QCD: I. Strategy and tree-level study
}

\section{$\overline{\mathrm{F}}$ Collaboration}

\section{Michele Della Morte, ${ }^{a}$ Samantha Dooling, ${ }^{b, 1}$ Jochen Heitger, $^{b}$ Dirk Hesse ${ }^{c}$ and Hubert Simma ${ }^{d}$}

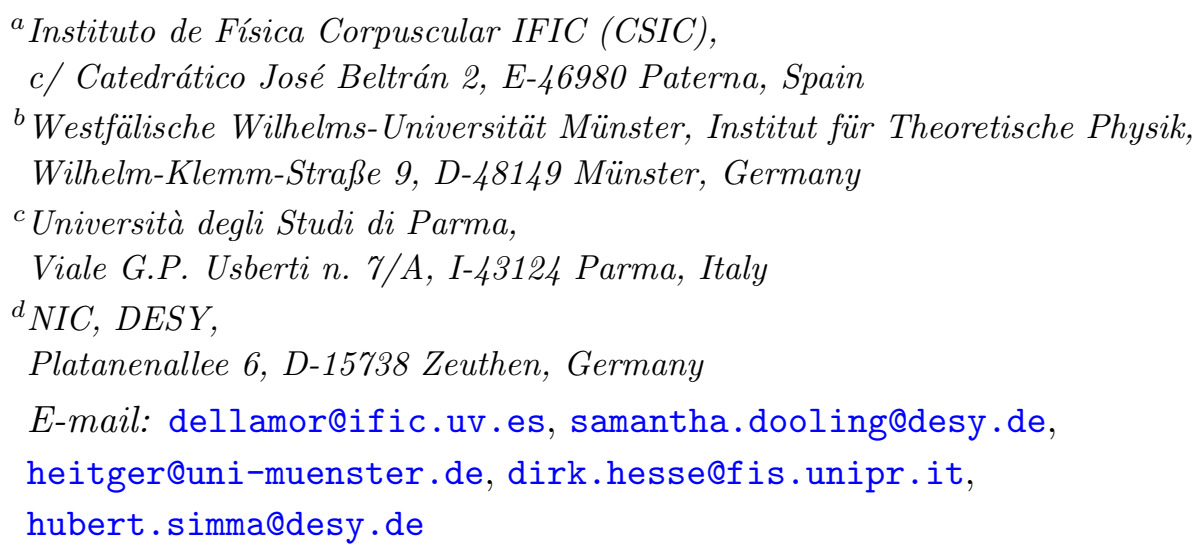

ABSTRACT: We present a strategy how to match the full set of components of the heavylight axial and vector currents in Heavy Quark Effective Theory (HQET), up to and including $1 / m_{\mathrm{h}}$-corrections, to QCD. While the ultimate goal is to apply these matching conditions non-perturbatively, in this study we first have implemented them at tree-level, in order to find good choices of the matching observables with small $\mathcal{O}\left(1 / m_{\mathrm{h}}^{2}\right)$ contributions. They can later be employed in the non-perturbative matching procedure which is a crucial part of precision HQET computations of semileptonic decay form factors in lattice QCD.

KEYwords: Lattice Gauge Field Theories, B-Physics, Heavy Quark Physics

ARXiv EPRINT: 1312.1566

\footnotetext{
${ }^{1}$ Present address: Deutsches Elektronen-Synchrotron DESY, Notkestraße 85, D-22607 Hamburg, Germany.
} 


\section{Contents}

1 Introduction 1

2 General form of the matching equations and strategy 4

$\begin{array}{ll}2.1 \text { Heavy-light axial current in HQET } & 6\end{array}$

3 Matching of heavy-light currents at $\mathcal{O}\left(1 / m_{\mathrm{h}}\right) \quad 8$

3.1 Definition of the correlation functions in QCD 8

$\begin{array}{ll}3.2 \text { Choice of the observables } & 10\end{array}$

$\begin{array}{lll}3.3 & \text { HQET parameters of the Lagrangian and of the axial current } & 12\end{array}$

4 Tree-level results for heavy-light currents at $\mathcal{O}\left(1 / m_{\mathrm{h}}\right) \quad 14$

5 Conclusions 18

A Explict form of the matching matrix for $\mathscr{L}^{\mathrm{HQET}}, A_{0}$ and $A_{k} \quad 21$

B Correlation functions and observables for the matching of the vector $\begin{array}{ll}\text { current } & 27\end{array}$

\section{Introduction}

Experimental and theoretical studies of B-meson decays belong to the major activities of the particle physics community within the realm of indirect searches for physics beyond the Standard Model. In recent years, experimental advances have led to high-precision B-factory experiments and $\mathrm{LHCb}$, with the potential to observe signatures of New Physics by unveiling contributions to those weak decays owing to virtual effects of particles not present in the Standard Model. These effects are known to be small and possibly quantifiable through the elements of the CKM matrix. Its crucial ingredients are measured decay branching ratios on the experimental side and low-energy hadron matrix elements encoding the strong interaction effects on the theory side. At the current high level of experimental precision, the rôle of equally accurate predictions from theory with controlled errors becomes increasingly important. Lattice QCD provides an ab-initio non-perturbative approach for a reliable and precise evaluation of these hadronic matrix elements.

In the B-meson sector, a prominent example is the entry $\left|V_{\mathrm{ub}}\right|$ of the CKM matrix, which can be estimated independently from the leptonic $\mathrm{B} \rightarrow \tau \nu$ and the exclusive semileptonic $\mathrm{B} \rightarrow \pi l \nu$ decay. It has received much attention in the past, since indications for a tension at the level of about $3 \sigma$ between these two determinations have been reported (see, e.g., refs. [1-4] and therein). Over the last few years, the ALPHA Collaboration has devised and implemented a non-perturbative strategy for the computation of phenomenologically 
relevant B-physics parameters in the framework of lattice Heavy Quark Effective Theory (HQET) [5-10]. This strategy separates the b-quark mass scale from the other intrinsic scales in a lattice simulation by a systematic expansion in the inverse heavy quark mass, $1 / m_{\mathrm{h}}$, and is able to subtract the cumbersome ultraviolet power divergences of the effective theory (due to operator mixing under renormalization) such that the continuum limit exists. While the low-energy constants of the effective theory (called HQET parameters from now on) entering the Lagrangian and the time component of the heavy-light axial-vector current at order $1 / m_{\mathrm{h}}$ have already been determined non-perturbatively $[7,10]$ and applied to phenomenology $[11,12]$, it is a natural and instructive next step to extend our HQET programme to also cover all components of the weak heavy-light axial and vector currents. In particular, the QCD matrix elements of the vector current between $\mathrm{B}\left(\mathrm{B}_{\mathrm{S}}\right)$ and $\pi(\mathrm{K})$ states at finite momenta, entering the semileptonic processes $\mathrm{B} \rightarrow \pi l \nu$ and $\mathrm{B}_{\mathrm{s}} \rightarrow \mathrm{K} l \nu$, respectively, are parameterized by two form factors to be predicted by non-perturbative QCD and HQET. Hence, determining the HQET parameters associated with the vector current including its $1 / m_{\mathrm{h}}$-corrections will be an essential prerequisite for our current attempt at computing for the first time these semileptonic B-decay form factors by means of lattice HQET at $\mathcal{O}\left(1 / m_{\mathrm{h}}\right)[13-15]$.

The purpose of the present paper is twofold. We first formulate a set of possible matching conditions for the HQET parameters appearing in the Lagrangian and in all components of the heavy-light axial $\left(A_{\mu}\right)$ and vector $\left(V_{\mu}\right)$ currents including all $1 / m_{\mathrm{h}^{-}}$ terms, which actually comprises terms of mass dimension five in the action and dimension four in the currents. This results in a total of 19 HQET parameters and thus at least 19 matching equations required to determine them. The second goal is to gain some insight into the intrinsic ambiguity of the chosen matching conditions and to try to reduce that to a suitable level. Let us expand on this point. Each matching condition amounts to evaluate a certain observable in QCD and in HQET (including $1 / m_{\mathrm{h}}$-terms) and to equate one with the other, in order to define the parameters in HQET. Ideally, one would like each observable to receive negligible contributions from $\mathcal{O}\left(1 / m_{\mathrm{h}}^{2}\right)$ terms and if possible to be sensitive to a single parameter only. Otherwise, unnaturally large $\mathcal{O}\left(1 / m_{\mathrm{h}}^{2}\right)$ contributions may propagate into the physical quantities, which one ultimately wants to compute in largevolume HQET after the matching step. Indeed, as in previous applications of our general strategy, the matching is here performed in finite volume with Schrödinger functional (SF) boundary conditions and linear extent $L$ [16-19]. Hence, our criterion for an optimal choice of matching observables is to have them such that $\left(L m_{\mathrm{h}}\right)^{-n}$-corrections with $n \geq 2$ can be neglected. These observables will then be considered as good candidates for the envisaged non-perturbative matching computation by numerical simulations.

We investigate in this paper the full system of 19 matching equations at tree-level of perturbation theory, where the HQET parameters are known exactly and the solution of the system can be studied as a function of $m_{\mathrm{h}}$ (and of further kinematical variables). In this way we can estimate the size of $1 / m_{\mathrm{h}}^{2}$-contributions in the matching observables and in the HQET parameters. The observables considered here are constructed from SF two- and three-point functions with appropriate kinematics. For a one-loop study of the renormalization factors of the static currents $A_{k}$ and $V_{0}$ based on three-point functions, we refer to the companion paper [20]. 
The ALPHA Collaboration's B-physics programme is based on a non-perturbative matching of HQET to QCD in finite volume. Let us briefly recall it for later convenience. The HQET Lagrangian

$$
\mathscr{L}^{\mathrm{HQET}}(x)=\mathscr{L}^{\text {stat }}(x)+m_{\text {bare }} \bar{\psi}_{\mathrm{h}}(x) \psi_{\mathrm{h}}(x)-\omega_{\mathrm{kin}} O_{\text {kin }}(x)-\omega_{\text {spin }} O_{\text {spin }}(x),
$$

at leading order in $1 / m_{\mathrm{h}}$ is just the (static) term

$$
\mathscr{L}^{\text {stat }}(x)=\bar{\psi}_{\mathrm{h}}(x) D_{0} \psi_{\mathrm{h}}(x)
$$

(plus the mass term that only leads to a shift of the energy levels). At order $1 / m_{\mathrm{h}}$, two additional interaction terms are included

$$
O_{\text {kin }}(x)=\bar{\psi}_{\mathrm{h}}(x) \mathbf{D}^{2} \psi_{\mathrm{h}}(x), \quad O_{\text {spin }}(x)=\bar{\psi}_{\mathrm{h}}(x) \boldsymbol{\sigma} \cdot \mathbf{B} \psi_{\mathrm{h}}(x) .
$$

They represent the kinetic energy from heavy quark's residual motion and the chromomagnetic interaction with the gluon field. $\mathbf{D}^{2}, \mathbf{B}$, and $D_{0}$ are defined in ref. [7]. Thus, the HQET Lagrangian has three parameters: $m_{\text {bare }}, \omega_{\text {kin }}$, and $\omega_{\text {spin }}$. The predictivity of the effective theory is only granted, once these HQET parameters have been fixed by a nonperturbative matching to QCD $[5,21]$ : three (properly renormalized) QCD observables, $\Phi_{i}$ $(i=1,2,3)$, evaluated in the continuum limit of finite-volume $Q C D$, are matched to their counterparts computed in HQET by imposing

$$
\Phi_{i}^{\mathrm{QCD}}\left(L, m_{\mathrm{h}}, 0\right)=\Phi_{i}^{\mathrm{HQET}}\left(L, m_{\mathrm{h}}, a\right),
$$

for any value of the lattice spacing $a$. While the l.h.s. of this "matching" equation is defined in the continuum,

$$
\Phi_{i}^{\mathrm{QCD}}\left(L, m_{\mathrm{h}}, 0\right)=\lim _{a \rightarrow 0} \Phi_{i}^{\mathrm{QCD}}\left(L, m_{\mathrm{h}}, a\right),
$$

the quantities $\Phi_{i}^{\mathrm{HQET}}$ are understood to be expanded up to a given order in $1 / m_{\mathrm{h}}$ (NLO in our setup) and computed in HQET at a finite lattice spacing. By solving this system of (at this point three) matching equations, the resulting HQET parameters become functions of $m_{\mathrm{h}}$ and $a$ and can be pushed to lattice spacings for use in phenomenological applications with large-volume simulations by step-scaling methods. For more details, the reader may consult, e.g., refs. [5, 7, 10, 22], where this programme has been completed in the quenched approximation and for two flavours of $\mathcal{O}(a)$ improved Wilson fermions.

For many relevant phenomenological applications in heavy quark physics, one also needs (correlation functions of) composite fields $O^{\mathrm{QCD}}(x)$, which are local combinations of the fundamental fields. The corresponding effective operators typically represent electroweak or other non-QCD interactions. In HQET such operators are written as linear combinations

$$
O^{\mathrm{HQET}}\left(m_{\mathrm{h}}\right)=Z_{O}\left\{O^{\text {stat }}+\sum c_{n} O_{n}\right\}=O^{\mathrm{QCD}}\left(m_{\mathrm{h}}\right)+\mathcal{O}\left(1 / m_{\mathrm{h}}^{2}\right),
$$

where the equality is meant for matrix elements of corresponding states in the fundamental (QCD) and effective (HQET) theories. The r.h.s. of the first equation above in general 
requires to include all operators which have a mass dimension one higher than $O^{\mathrm{QCD}}$ (or $O^{\text {stat }}$ ) and which transform in the same way as $O^{\mathrm{QCD}}$ under the common set of symmetries of QCD and HQET (but linearly independent with respect to the equations of motion of the effective theory). The operators $O_{n}$ are needed for the renormalization and $\mathcal{O}(a)$ improvement of the effective theory, and in order to systematically include in HQET the $m_{\mathrm{h}^{-}}$ effects of QCD. These are encoded into the non-trivial $m_{\mathrm{h}}$-dependence of the parameters $Z_{O}$ and $c_{n}$ in eq. (1.6). The remaining $m_{\mathrm{h}}$-dependence in $\Phi_{i}^{\mathrm{QCD}}\left(L, m_{\mathrm{h}}, 0\right)-\Phi_{i}^{\mathrm{HQET}}\left(L, m_{\mathrm{h}}, a\right)$ is useful to quantify higher-order (in $1 / m_{\mathrm{h}}$ ) corrections. These will be studied here at tree-level for a particular class of matching conditions. In the following we assume all light quark masses to be set to zero, and we shall only consider heavy-light currents, $O=J \in\left\{A_{0}, A_{k}, V_{0}, V_{k}\right\}$, but the general strategy can straightforwardly be applied to other operators, such as four-quark operators. The HQET expansions of the currents at $\mathcal{O}\left(1 / m_{\mathrm{h}}\right)$ requires 16 additional HQET parameters. As it will be worked out in the next sections, they can be fixed on a similar footing as outlined for the 3 parameters of the Lagrangian above. Note that the systematic expansion in $1 / m_{\mathrm{h}}$ is part of the very definition of HQET and renders it order by order in $1 / m_{\mathrm{h}}$ a renormalizable field theory [5].

The plan of the paper is as follows. In section 2 we explain the general structure of the system of matching equations for the parameters of the HQET Lagrangian together with heavy-light currents and illustrate it for the case of the axial current as a representative example. Section 3 introduces the full set of matching observables as built from suitable finite-volume SF correlation functions and summarizes their generic HQET expansions that enter the matching step; explicit expressions of the expansion coefficients are given in appendix A. Our detailed discussion in the main text is focused on the case of the axial current and the corresponding formulae for the vector current are collected in appendix B. In section 4 our results for the HQET parameters from the tree-level matching are discussed, and, based on that, we advocate our preferred choice of observables for the non-perturbative matching procedure in the future. Section 5 contains our conclusions.

\section{General form of the matching equations and strategy}

In order to determine all parameters, which occur at order $1 / m_{\mathrm{h}}$ in the HQET expansion (eq. (1.6)) of a current $J$, the matching conditions in eq. (1.4) have to be solved for a corresponding number of observables $\Phi_{i}$ (say $i \in I_{J}$ ).

These observables are constructed from suitable combinations of correlation functions, $C_{J}$, which typically have a single insertion of $J$. If the HQET expansion of $J$ is written as

$$
J^{\mathrm{HQET}}=Z_{J}^{\mathrm{HQET}}\left\{J^{\mathrm{stat}}+\sum_{n} c_{J_{n}} J_{n}\right\}+\mathcal{O}\left(1 / m_{\mathrm{h}}^{2}\right),
$$

the HQET expansion of the correlation functions has the generic form

$$
C_{J}^{\mathrm{HQET}}=Z_{J}^{\mathrm{HQET}} Z_{C}^{\mathrm{HQET}} e^{-m_{\mathrm{bare}} x_{C}}\left\{C_{J}^{\mathrm{stat}}+\omega_{\mathrm{kin}} C_{J}^{\mathrm{kin}}+\omega_{\mathrm{spin}} C_{J}^{\mathrm{spin}}+\sum_{n} c_{J_{n}} C_{J_{n}}^{1 / \mathrm{m}}\right\}
$$


where all correlators on the r.h.s. are computed in the static approximation. The only place where the parameter $m_{\text {bare }}$ appears is the factor $e^{-m_{\text {bare }} x_{C}}$, with $x_{C}$ related to the time distances of the heavy (static) quark fields entering in $C_{J}^{\mathrm{HQET}}$. Aside from $Z_{J}^{\mathrm{HQET}}$, all other (wave-function) renormalization factors contributing to $C_{J}^{\mathrm{HQET}}$ are collected in $Z_{C}^{\mathrm{HQET}}$.

In the correlation functions on the r.h.s. of eq. (2.2), the leading-order term $C_{J}^{\text {stat }}$ has just one insertion of $J^{\text {stat }}\left(\right.$ instead of $J$ ), while $C_{J}^{\text {kin/spin }} \operatorname{differ}$ from $C_{J}^{\text {stat }}$ by an extra insertion (summed over the entire space-time volume) of the $1 / m_{\mathrm{h}}$-terms $O_{\text {kin }}$ or $O_{\text {spin }}$ from the Lagrangian. The other next-to-leading contributions $C_{J_{n}}^{1 / \mathrm{m}}$ have an insertion of one of the higher-dimensional operators $J_{n}$ from the expansion in eq. (2.1).

The observables $\Phi_{i}$ are then defined as suitable combinations of such correlation functions (e.g., logarithms of ratios, see section 3 for explicit definitions). Thus, the renormalization factor $Z_{C}^{\mathrm{HQET}}$ cancels, while a possibly remaining factor $Z_{J}^{\mathrm{HQET}}$ needs to be expanded in $1 / m_{\mathrm{h}}$ in order to consistently keep only terms up to order $1 / m_{\mathrm{h}}$ in eq. (2.2). Moreover, only one of the observables, say $\Phi_{1}$, is left with an explicit dependence on $m_{\text {bare }}$.

By combining all HQET parameters into a vector

$$
\omega=\left(m_{\text {bare }}, \omega_{\text {kin }}, \omega_{\text {spin }}, c_{J_{1}}, \ldots, \ln Z_{J}^{\mathrm{HQET}}, \ldots\right)^{T},
$$

the HQET expansion of the observables can be written in the compact form

$$
\Phi_{i}^{\mathrm{HQET}}(L, M, a)=\eta_{i}(L, a)+\varphi_{i}^{j}(L, a) \omega_{j}(M, a)+\mathcal{O}\left(1 / m_{\mathrm{h}}^{2}\right),
$$

where $M$ is the Renormalization Group Invariant (RGI) heavy quark mass and the vector $\eta$ accounts for the contribution of the static terms $C_{J}^{\text {stat }}$ in the correlators involved. As mentioned in the introduction, because the matching is performed at a finite value of the renormalized quark mass of QCD, the parameters get a non-trivial $M$-dependence even when working only in the static approximation of HQET. Note that in general and non-perturbatively, only the combination of HQET quantities, which enters on the r.h.s. of eq. (2.4), is expected to have a continuum limit. The tree-level approximation is exceptional in this respect as each individual term on the r.h.s. has a well defined continuum limit in that case.

The matrix $\varphi_{i}^{j}$ of eq. (2.4) reflects the structure of the matching equations (1.4). To illustrate its general structure, we group the parameters into blocks $\left(m_{\text {bare }}\right),\left(\omega_{\text {kin }}, \omega_{\text {spin }}\right)$, $\left(c_{J_{i}}, Z_{J}\right),\left(c_{J_{i}^{\prime}}, Z_{J^{\prime}}\right),\left(c_{J_{i}^{\prime \prime}}, Z_{J^{\prime \prime}}\right), \ldots$, and assume that $J$ is the current which is used in $\Phi_{1}$. A suitable choice of the other observables then leads to the following natural form of the matrix $\varphi_{i}^{j}$ :

- In the first column all entries, except for $\varphi_{1}^{1}$, vanish.

- The first row has non-vanishing $\varphi_{1}^{2}$ and $\varphi_{1}^{3}$ corresponding to contributions from $\omega_{\text {kin }}$ and $\omega_{\text {spin }}$ to $\Phi_{1}$. In addition, there may be non-zero $\varphi_{1}^{j}$ with $j$ from a single block, which corresponds to the current used in $\Phi_{1}$. In our case, this is due to the $A_{0,1}$-term, which enters in our matching condition for $m_{\text {bare }}$ (see later). However, it is easy to show that the corresponding $\varphi_{1}^{4}$ would vanish in the large- $L$ limit. 
- The rest has a simple block structure, with non-zero blocks only in the second blockcolumn (corresponding to contributions from $\omega_{\text {kin }}$ and $\omega_{\text {spin }}$ ) and in the blocks on the diagonal (corresponding to the mixings in the last term of eq. (2.2)).

Thus, we schematically have the following block structure:

$$
\varphi=\left(\begin{array}{c|c|c|c|c}
\varphi_{1}^{1} & * & * & 0 & 0 \\
\hline 0 & * & 0 & 0 & 0 \\
\hline 0 & * & * & 0 & 0 \\
\hline 0 & * & 0 & * & 0 \\
\hline 0 & * & 0 & 0 & *
\end{array}\right) .
$$

Each time an additional (effective) operator $J^{\prime}$ is included, a new set of observables $\Phi_{i}$ with $i \in I_{J^{\prime}}$ can be added such that $\varphi_{i}^{j}$, and hence the matching equations, have the above block structure. The system can therefore always be solved simply by block-wise backward substitution in order to determine the HQET parameters.

\subsection{Heavy-light axial current in HQET}

As an example of this general structure of the matching equations, we recall the explicit form of the (renormalized) heavy-light axial current in HQET. The time component is

$$
A_{0}^{\mathrm{HQET}}(x)=Z_{\mathrm{A}_{0}}^{\mathrm{HQET}}\left[A_{0}^{\mathrm{stat}}(x)+\sum_{i=1}^{2} c_{\mathrm{A}_{0, i}} A_{0, i}(x)\right],
$$

with the leading-order (static) term

$$
A_{\mu}^{\text {stat }}(x)=\bar{\psi}_{\ell}(x) \gamma_{\mu} \gamma_{5} \psi_{\mathrm{h}}(x)
$$

and two additional dimension-four contributions

$$
\begin{aligned}
& A_{0,1}(x)=\bar{\psi}_{\ell}(x) \frac{1}{2} \gamma_{5} \gamma_{i}\left(\nabla_{i}^{\mathrm{S}}-\overleftarrow{\nabla}_{i}^{\mathrm{S}}\right) \psi_{\mathrm{h}}(x), \\
& A_{0,2}(x)=\bar{\psi}_{\ell}(x) \frac{1}{2} \gamma_{5} \gamma_{i}\left(\nabla_{i}^{\mathrm{S}}+\overleftarrow{\nabla}_{i}^{\mathrm{S}}\right) \psi_{\mathrm{h}}(x),
\end{aligned}
$$

where all derivatives are symmetric,

$$
\widetilde{\partial}_{i}=\frac{1}{2}\left(\partial_{i}+\partial_{i}^{*}\right), \quad \overleftarrow{\nabla}_{i}^{\mathrm{S}}=\frac{1}{2}\left(\overleftarrow{\nabla}_{i}+\overleftarrow{\nabla}_{i}^{*}\right), \quad \nabla_{i}^{\mathrm{S}}=\frac{1}{2}\left(\nabla_{i}+\nabla_{i}^{*}\right)
$$

A comment on the definition of the covariant derivatives is in order here. On a finite lattice, the action of the covariant derivative on a fermion field is given by

$$
\begin{aligned}
\nabla_{\mu} \psi(x) & =\frac{1}{a}\left[\lambda_{\mu} U(x, \mu) \psi(x+a \hat{\mu})-\psi(x)\right], \\
\nabla_{\mu}^{*} \psi(x) & =\frac{1}{a}\left[\psi(x)-\lambda_{\mu}^{-1} U(x-a \hat{\mu}, \mu)^{-1} \psi(x-a \hat{\mu}],\right.
\end{aligned}
$$

where $\hat{\mu}$ is a unit vector in direction $\mu$. The left action is defined as

$$
\begin{aligned}
\bar{\psi}(x) \overleftarrow{\nabla}_{\mu} & =\frac{1}{a}\left[\bar{\psi}(x+a \hat{\mu}) U(x, \mu)^{-1} \lambda_{\mu}^{-1}-\bar{\psi}(x)\right], \\
\bar{\psi}(x) \overleftarrow{\nabla}_{\mu}^{*} & =\frac{1}{a}\left[\bar{\psi}(x)-\bar{\psi}(x-a \hat{\mu}) U(x-a \hat{\mu}, \mu) \lambda_{\mu}\right] .
\end{aligned}
$$


We want to emphasize here the appearance of the phase factor

$$
\lambda_{\mu}=e^{i a \theta_{\mu} / L}, \quad \theta_{0}=0, \quad-\pi<\theta_{k} \leq \pi,
$$

which plays an important rôle in the following, as it will be used to vary the kinematics in the correlation functions. Up to an Abelian gauge transformation, it is equivalent to imposing the generalized periodic boundary conditions

$$
\psi(x+L \hat{k})=e^{i \theta_{k}} \psi(x), \quad \bar{\psi}(x+L \hat{k})=\bar{\psi}(x) e^{-i \theta_{k}} .
$$

In our setup, this phase, sometimes referred to as twisting [23], can be employed to inject a momentum $\left|\vec{\theta}_{\mathrm{h}}-\vec{\theta}_{\ell}\right| / L$ in the correlation functions we describe in the next section. It is clear that for $\vec{\theta}_{\mathrm{h}}=\vec{\theta}_{\ell}$ composite fields as $A_{0,2}(x)$ above can be associated with total derivative operators. In such a case their contribution vanishes once inserted in correlation functions, unless a non-zero total momentum component (integer multiple of $2 \pi / L$ ) is explicitly considered by introducing spatial integrations with the appropriate Fourier factors (which we avoid to do, in order to fully exploit volume averaging in view of numerical applications).

Concerning the spatial components of the axial-vector current, their HQET expansion (see ref. [22]) can be written as

$$
A_{k}^{\mathrm{HQET}}(x)=Z_{\overrightarrow{\mathrm{A}}}^{\mathrm{HQET}}\left[A_{k}^{\mathrm{stat}}(x)+\sum_{i=1}^{4} c_{\mathrm{A}_{k, i}} A_{k, i}(x)\right],
$$

with the following four extra terms:

$$
\begin{aligned}
& A_{k, 1}(x)=\bar{\psi}_{\ell}(x) \frac{1}{2}\left(\nabla_{i}^{\mathrm{S}}-\overleftarrow{\nabla}_{i}^{\mathrm{S}}\right) \gamma_{i} \gamma_{5} \gamma_{k} \psi_{\mathrm{h}}(x) \\
& A_{k, 2}(x)=\bar{\psi}_{\ell}(x) \frac{1}{2}\left(\nabla_{k}^{\mathrm{S}}-\overleftarrow{\nabla}_{k}^{\mathrm{S}}\right) \gamma_{5} \psi_{\mathrm{h}}(x) \\
& A_{k, 3}(x)=\bar{\psi}_{\ell}(x) \frac{1}{2}\left(\nabla_{i}^{\mathrm{S}}+\overleftarrow{\nabla}_{i}^{\mathrm{S}}\right) \gamma_{i} \gamma_{5} \gamma_{k} \psi_{\mathrm{h}}(x) \\
& A_{k, 4}(x)=\bar{\psi}_{\ell}(x) \frac{1}{2}\left(\nabla_{k}^{\mathrm{S}}+\overleftarrow{\nabla}_{k}^{\mathrm{S}}\right) \gamma_{5} \psi_{\mathrm{h}}(x) .
\end{aligned}
$$

The vector current components are just obtained by dropping $\gamma_{5}$ in these expressions and by changing $c_{\mathrm{A}_{\mu, i}} \rightarrow c_{\mathrm{V}_{\mu, i}}$. The classical values of the coefficients are

$$
c_{\mathrm{A}_{0,1}}=c_{\mathrm{A}_{0,2}}=-c_{\mathrm{A}_{k, 1}}=-c_{\mathrm{A}_{k, 3}}=-\frac{1}{2 m_{\mathrm{h}}}, \quad \text { whilst } \quad c_{\mathrm{A}_{k, 2}}=c_{\mathrm{A}_{k, 4}}=\frac{1}{m_{\mathrm{h}}},
$$

and analogous for the vector current, apart from $c_{\mathrm{V}_{0,1}}, c_{\mathrm{V}_{0,2}}$ and $c_{\mathrm{V}_{k, 2}}, c_{\mathrm{V}_{k, 4}}$ which differ by a sign. As a remark, the heavy-light pseudoscalar and scalar densities $P$ and $S$ are given exactly by the same expressions as $V_{0}$ and $A_{0}$ (due to the $\gamma_{0} \psi_{\mathrm{h}}=\psi_{\mathrm{h}}$ property) with the changes $c_{\mathrm{A}_{0, i}} \rightarrow c_{\mathrm{P}_{i}}, c_{\mathrm{V}_{0, i}} \rightarrow c_{\mathrm{S}_{i}}$. Such coefficients can be determined essentially through the same matching conditions we are going to implement for $A_{0}$ and $V_{0}$, using instead correlation functions involving $P$ and $S$ on the QCD side. 
We note in passing that the mixing of $A_{k}^{\text {stat }}$ with $A_{k, 2}$ and $A_{k, 4}$ is due to the breaking of spin-symmetry by $O_{\text {spin }}$. Similarly, the mixing of the static currents with the (combinations of) operators, like $A_{0,1}+A_{0,2}, A_{k, 1}+A_{k, 3}$, and $A_{k, 2}+A_{k, 4}$, where the derivative only acts on the heavy quark, are due to the breaking of local heavy-flavour conservation by $O_{\text {kin }}$.

In refs. [7, 10] only the time component of the axial current has been considered, and $A_{0,2}$ has not been included because it does not contribute to correlation functions at (total) zero momentum, such as those typically used to compute decay constants. The corresponding 5 HQET parameters can then be determined from a restricted system of matching equations which has the form (see ref. [7] for a precise definition of the quantities in the matrix below)

$$
\left(\begin{array}{c}
L \Gamma^{\mathrm{P}} \\
\hline R_{1} \\
\frac{3}{4} \ln \left(\frac{f_{1}}{k_{1}}\right) \\
\hline R_{\mathrm{A}} \\
\ln \left(\frac{-f_{\mathrm{A}}}{\sqrt{f_{1}}}\right)
\end{array}\right)=\left(\begin{array}{c}
L \Gamma^{\mathrm{stat}} \\
\hline R_{1}^{\mathrm{stat}} \\
0 \\
\hline R_{\mathrm{A}}^{\text {stat }} \\
\zeta_{\mathrm{A}}
\end{array}\right)+\left(\begin{array}{c|cc|cc}
L & L \Gamma^{\mathrm{kin}} & L \Gamma^{\mathrm{spin}} & L \Gamma_{\delta \mathrm{A}} & 0 \\
\hline 0 & R_{1}^{\mathrm{kin}} & 0 & 0 & 0 \\
0 & 0 & \rho_{1}^{\mathrm{spin}} & 0 & 0 \\
\hline 0 & R_{\mathrm{A}}^{\mathrm{kin}} & R_{\mathrm{A}}^{\mathrm{spin}} & R_{\delta \mathrm{A}} & 0 \\
0 & \psi^{\mathrm{kin}} & \psi^{\mathrm{spin}} & \rho_{\delta \mathrm{A}} & 1
\end{array}\right) \cdot\left(\begin{array}{c}
m_{\text {bare }} \\
\hline \omega_{\text {kin }} \\
\omega_{\text {spin }} \\
\hline a c_{\mathrm{A}}^{(1)} \\
\ln Z_{\mathrm{A}}^{\mathrm{HQET}}
\end{array}\right) .
$$

\section{Matching of heavy-light currents at $\mathcal{O}\left(1 / m_{\mathrm{h}}\right)$}

We now consider the full system of 19 matching equations for HQET including the currents $J=A_{0}, A_{k}, V_{0}, V_{k}$. We arrange the parameters in a vector $\omega \equiv \omega_{i}$ as follows:

\begin{tabular}{|lll|}
\hline$i$ & $\omega_{i}$ & origin \\
\hline $1,2,3$ & $m_{\text {bare }}, \omega_{\text {kin }}, \omega_{\text {kin }}$ & $\mathscr{L}^{\mathrm{HQET}}$ \\
$4, \ldots, 6$ & $c_{\mathrm{A}_{0,1}}, c_{\mathrm{A}_{0,2}}, \ln Z_{A_{0}}^{\mathrm{HQET}}$ & $A_{0}^{\mathrm{HQET}}$ \\
$7, \ldots, 11$ & $c_{\mathrm{A}_{k, 1}}, c_{\mathrm{A}_{k, 2}}, c_{\mathrm{A}_{k, 3}}, c_{\mathrm{A}_{k, 4}}, \ln Z_{\overrightarrow{\mathrm{A}}}^{\mathrm{HQET}}$ & $A_{k}^{\mathrm{HQET}}$ \\
$12 \ldots, 14$ & $c_{\mathrm{V}_{0,1}}, c_{\mathrm{V}_{0,2}}, \ln Z_{V_{0}}^{\mathrm{HQET}}$ & $V_{0}^{\mathrm{HQET}}$ \\
$15, \ldots, 19$ & $c_{\mathrm{V}_{k, 1}}, c_{\mathrm{V}_{k, 2}}, c_{\mathrm{V}_{k, 3}}, c_{\mathrm{V}_{k, 4}}, \ln Z_{\overrightarrow{\mathrm{V}}}^{\mathrm{HQET}}$ & $V_{k}^{\mathrm{HQET}}$ \\
\hline
\end{tabular}

where in the last column we have indicated for each parameter, whether it enters the HQET Lagrangian or the expansion of a current component. We limit our more detailed discussion to the parameters in $\mathscr{L}^{\mathrm{HQET}}, A_{0}$ and $A_{k}$ (i.e., $\omega_{i}$ with $1 \leq i \leq 11$ ). The matching equations for $V_{0}$ and $V_{k}$ are simply obtained by generalizing those for $A_{0}$ and $A_{k}$, and further details can be found in appendix B.

\subsection{Definition of the correlation functions in QCD}

As in previous work $[5,7,10]$, we define the matching observables in the SF with homogeneous boundary conditions at $x_{0}=0$ and $x_{0}=T$ [16-18]. Correlation functions can be 
formed from composite fields in the bulk, $0<x_{0}<T$, and boundary quark fields. We obviously think of $m_{\mathrm{h}}$ being around the mass of the b-quark and use the label $\mathrm{b}$ to refer to heavy relativistic quarks. The subscript I of the currents indicates that they are $\mathcal{O}(a)$ improved, as defined in ref. [24, 25] for Wilson quarks. We define in QCD on the lattice:

- Boundary-to-boundary correlators

$$
\begin{aligned}
F_{1}\left(\vec{\theta}_{\ell}, \vec{\theta}_{\mathrm{b}}\right) & =-\frac{a^{12}}{2 L^{6}} \sum_{\mathbf{u}, \mathbf{v}, \mathbf{y}, \mathbf{z}}\left\langle\bar{\zeta}_{\ell}^{\prime}(\mathbf{u}) \gamma_{5} \zeta_{\mathrm{b}}^{\prime}(\mathbf{v}) \bar{\zeta}_{\mathrm{b}}(\mathbf{y}) \gamma_{5} \zeta_{\ell}(\mathbf{z})\right\rangle, \\
K_{1}\left(\vec{\theta}_{\ell}, \vec{\theta}_{\mathrm{b}}\right) & =-\frac{a^{12}}{6 L^{6}} \sum_{k} \sum_{\mathbf{u}, \mathbf{v}, \mathbf{y}, \mathbf{z}}\left\langle\bar{\zeta}_{\ell}^{\prime}(\mathbf{u}) \gamma_{k} \zeta_{\mathrm{b}}^{\prime}(\mathbf{v}) \bar{\zeta}_{\mathrm{b}}(\mathbf{y}) \gamma_{k} \zeta_{\ell}(\mathbf{z})\right\rangle, \\
K_{1}^{\ell \ell}\left(\vec{\theta}_{\ell}, \vec{\theta}_{\ell^{\prime}}\right) & =-\frac{a^{12}}{6 L^{6}} \sum_{k} \sum_{\mathbf{u}, \mathbf{v}, \mathbf{y}, \mathbf{z}}\left\langle\bar{\zeta}_{\ell}^{\prime}(\mathbf{u}) \gamma_{k} \zeta_{\ell^{\prime}}^{\prime}(\mathbf{v}) \bar{\zeta}_{\ell^{\prime}}(\mathbf{y}) \gamma_{k} \zeta_{\ell}(\mathbf{z})\right\rangle . \\
J_{\mathrm{A}_{1}}^{1}\left(x_{0}, \vec{\theta}_{\ell}, \vec{\theta}_{\ell^{\prime}}, \vec{\theta}_{\mathrm{b}}\right) & =-\frac{a^{15}}{2 L^{6}} \sum_{\mathbf{u}, \mathbf{v}, \mathbf{y}, \mathbf{z}, \mathbf{x}}\left\langle\bar{\zeta}_{\ell^{\prime}}^{\prime}(\mathbf{u}) \gamma_{1} \zeta_{\ell}^{\prime}(\mathbf{v})\left(A_{\mathrm{I}}\right)_{1}(x) \bar{\zeta}_{\mathrm{b}}(\mathbf{z}) \gamma_{5} \zeta_{\ell^{\prime}}(\mathbf{y})\right\rangle .
\end{aligned}
$$

- Bulk-to-boundary correlators

$$
\begin{aligned}
f_{\mathrm{A}_{0}}\left(x_{0}, \vec{\theta}_{\ell}, \vec{\theta}_{\mathrm{b}}\right) & =-\frac{a^{6}}{2} \sum_{\mathbf{y}, \mathbf{z}}\left\langle\left(A_{\mathrm{I}}\right)_{0}(x) \bar{\zeta}_{\mathrm{b}}(\mathbf{y}) \gamma_{5} \zeta_{\ell}(\mathbf{z})\right\rangle, \\
f_{\overrightarrow{\mathrm{A}}}\left(x_{0}, \vec{\theta}_{\ell}, \vec{\theta}_{\mathrm{b}}\right) & =i \frac{a^{6}}{6} \sum_{k} \sum_{\mathbf{y}, \mathbf{z}}\left\langle\left(A_{\mathrm{I}}\right)_{k}(x) \bar{\zeta}_{\mathrm{b}}(\mathbf{y}) \gamma_{5} \zeta_{\ell}(\mathbf{z})\right\rangle, \\
k_{\mathrm{A}_{2}}^{1}\left(x_{0}, \vec{\theta}_{\ell}, \vec{\theta}_{\mathrm{b}}\right) & =i \frac{a^{6}}{2} \sum_{\mathbf{y}, \mathbf{z}}\left\langle\left(A_{\mathrm{I}}\right)_{2}(x) \bar{\zeta}_{\mathrm{b}}(\mathbf{y}) \gamma_{1} \zeta_{\ell}(\mathbf{z})\right\rangle,
\end{aligned}
$$

where for the last two correlators the $\vec{\theta}$ angles have to be chosen different from zero, at least in the $z$-direction, as otherwise they would vanish due to either rotation invariance or parity.

The inclusion of the static quarks has been discussed in ref. [19], to which we refer for the definition of the boundary quark fields $\zeta_{\mathrm{h}}$ and $\bar{\zeta}_{\mathrm{h}}$. Taking $f_{\mathrm{A}_{0}}$ as an example, we define the correlation functions that enter in the $1 / m_{\mathrm{h}}$-expansion of HQET:

$$
\begin{gathered}
f_{\mathrm{A}_{0}}^{\mathrm{stat}}\left(x_{0}, \vec{\theta}_{\ell}\right)=-\frac{a^{6}}{2} \sum_{\mathbf{y}, \mathbf{z}}\left\langle A_{0}^{\mathrm{stat}}(x) \bar{\zeta}_{\mathrm{h}}(\mathbf{y}) \gamma_{5} \zeta_{\ell}(\mathbf{z})\right\rangle, \\
f_{\mathrm{A}_{0}}^{\mathrm{spin}}\left(x_{0}, \vec{\theta}_{\ell}\right)=-\frac{a^{10}}{2} \sum_{\mathbf{y}, \mathbf{z}, w}\left\langle O_{\operatorname{spin}}(w) A_{0}^{\mathrm{stat}}(x) \bar{\zeta}_{\mathrm{h}}(\mathbf{y}) \gamma_{5} \zeta_{\ell}(\mathbf{z})\right\rangle, \\
f_{\mathrm{A}_{0}}^{\mathrm{kin}}\left(x_{0}, \vec{\theta}_{\ell}, \vec{\theta}_{\mathrm{h}}\right)=-\frac{a^{10}}{2} \sum_{\mathbf{y}, \mathbf{z}, w}\left\langle O_{\mathrm{kin}}(w) A_{0}^{\mathrm{stat}}(x) \bar{\zeta}_{\mathrm{h}}(\mathbf{y}) \gamma_{5} \zeta_{\ell}(\mathbf{z})\right\rangle, \\
f_{\mathrm{A}_{0, i}}\left(x_{0}, \vec{\theta}_{\ell}, \vec{\theta}_{\mathrm{h}}\right)=-\frac{a^{6}}{2} \sum_{\mathbf{y}, \mathbf{z}}\left\langle A_{0, i}(x) \bar{\zeta}_{\mathrm{h}}(\mathbf{y}) \gamma_{5} \zeta_{\ell}(\mathbf{z})\right\rangle .
\end{gathered}
$$


We will use an analogous notation for all the HQET correlators appearing in the expansion of the QCD correlators defined in eqs. (3.1)-(3.7) and in appendix B. For the renormalized correlator $\left[f_{\mathrm{A}_{0}}\right]_{\mathrm{R}}$, the HQET expansion eq. (2.2) including order $1 / m_{\mathrm{h}}$-terms reads

$$
\left[f_{\mathrm{A}_{0}}\right]_{\mathrm{R}}^{\mathrm{HQET}}=Z_{\mathrm{A}_{0}}^{\mathrm{HQET}} Z_{\zeta_{\mathrm{h}}} Z_{\zeta} e^{-m_{\mathrm{bare}} x_{0}}\left\{f_{\mathrm{A}_{0}}^{\mathrm{stat}}+\omega_{\text {kin }} f_{\mathrm{A}_{0}}^{\mathrm{kin}}+\omega_{\mathrm{spin}} f_{\mathrm{A}_{0}}^{\mathrm{spin}}+\sum_{i} c_{\mathrm{A}_{0, i}} f_{\mathrm{A}_{0, i}}\right\} .
$$

Notice that there are no $1 / m_{\mathrm{h}}$-terms arising from the HQET expansion of the boundary fields. These indeed vanish or can be absorbed into the field-normalization, either because of the equations of motion or by imposing the SF boundary condition

$$
P_{-} \zeta_{\mathrm{b}}=0, \quad P_{-}=\frac{1}{2}\left(1-\gamma_{0}\right)
$$

to all orders in $1 / m_{\mathrm{h}}$.

\subsection{Choice of the observables}

We now show that the correlators defined above are sufficient to form a set of rather simple observables, which can be employed to fix the HQET Lagrangian and the axial current components at $\mathcal{O}\left(1 / m_{\mathrm{h}}\right)$. We suppress the arguments $L, M$ and $a$ with respect to eq. (1.4) and rather emphasize the dependence on the fermionic periodicity angles entering the different correlators. For the determination of the parameters $\omega_{i}, 1 \leq i \leq 11$, we define the following 11 observables:

$$
\begin{array}{lll}
\Phi_{1}^{\mathrm{QCD}}\left(\vec{\theta}_{1}\right) & \equiv-L \cdot \tilde{\partial}_{0} \ln \left(-f_{\mathrm{A}_{0}}\left(x_{0}, \vec{\theta}_{1}, \vec{\theta}_{1}\right)\right), & \left(T=L, x_{0}=T / 2\right), \\
\Phi_{2}^{\mathrm{QCD}}\left(\vec{\theta}_{1}, \vec{\theta}_{2}\right) & \equiv \frac{1}{4} \ln \left(\frac{F_{1}\left(\vec{\theta}_{1}, \vec{\theta}_{1}\right)}{F_{1}\left(\vec{\theta}_{2}, \vec{\theta}_{2}\right)}\right)+\frac{3}{4} \ln \left(\frac{K_{1}\left(\vec{\theta}_{1}, \vec{\theta}_{1}\right)}{K_{1}\left(\vec{\theta}_{2}, \vec{\theta}_{2}\right)}\right),(T=L / 2), \\
\Phi_{3}^{\mathrm{QCD}}\left(\vec{\theta}_{1}\right) & \equiv \frac{3}{4} \ln \left(\frac{F_{1}\left(\vec{\theta}_{1}, \vec{\theta}_{1}\right)}{K_{1}\left(\vec{\theta}_{1}, \vec{\theta}_{1}\right)}\right), & (T=L / 2), \\
\Phi_{4}^{\mathrm{QCD}}\left(\vec{\theta}_{1}, \vec{\theta}_{2}\right) & \equiv \ln \left(\frac{f_{\mathrm{A}_{0}\left(x_{0}, \vec{\theta}_{1}, \vec{\theta}_{1}\right)}}{\left.f_{\mathrm{A}_{0}\left(x_{0}, \vec{\theta}_{2}, \vec{\theta}_{2}\right)}\right),}\right. & \left(T=L, x_{0}=T / 2\right), \\
\Phi_{5}^{\mathrm{QCD}}\left(\vec{\theta}_{1}, \vec{\theta}_{2}, \vec{\theta}_{3}\right) \equiv \ln \left(\frac{f_{\mathrm{A}_{0}}\left(x_{0}, \vec{\theta}_{1}, \vec{\theta}_{2}\right)}{\left.f_{\mathrm{A}_{0}\left(x_{0}, \vec{\theta}_{1}, \vec{\theta}_{3}\right)}\right)},\right. & \left(T=L, x_{0}=T / 2\right), \\
\Phi_{6}^{\mathrm{QCD}}\left(\vec{\theta}_{1}\right) & \equiv \ln \left(\frac{-f_{\mathrm{A}_{0}}\left(x_{0}, \vec{\theta}_{1}, \vec{\theta}_{1}\right)}{\left.\sqrt{F_{1}\left(\vec{\theta}_{1}, \vec{\theta}_{1}\right)}\right),}\right. & \left(T=L, x_{0}=T / 2\right),
\end{array}
$$




$$
\begin{array}{lll}
\Phi_{7}^{\mathrm{QCD}}\left(\vec{\theta}_{1}, \vec{\theta}_{2}\right) & \equiv \ln \left(\frac{f_{\overrightarrow{\mathrm{A}}}\left(x_{0}, \vec{\theta}_{1}, \vec{\theta}_{1}\right)}{f_{\overrightarrow{\mathrm{A}}}\left(x_{0}, \overrightarrow{\theta_{2}}, \overrightarrow{\theta_{2}}\right)}\right), & \left(T=L, x_{0}=T / 2\right), \\
\Phi_{8}^{\mathrm{QCD}}\left(\vec{\theta}_{1}, \vec{\theta}_{2}\right) & \equiv \ln \left(\frac{k_{\mathrm{A}_{2}}^{1}\left(x_{0}, \vec{\theta}_{1}, \vec{\theta}_{1}\right)}{k_{\mathrm{A}_{2}}^{1}\left(x_{0}, \overrightarrow{\theta_{2}}, \overrightarrow{\theta_{2}}\right)}\right), & \left(T=L, x_{0}=T / 2\right), \\
\Phi_{9}^{\mathrm{QCD}}\left(\vec{\theta}_{1}, \vec{\theta}_{2}, \vec{\theta}_{3}\right) \equiv \ln \left(\frac{f_{\overrightarrow{\mathrm{A}}}\left(x_{0}, \vec{\theta}_{1}, \vec{\theta}_{2}\right)}{f_{\overrightarrow{\mathrm{A}}}\left(x_{0}, \vec{\theta}_{1}, \vec{\theta}_{3}\right)}\right), & \left(T=L, x_{0}=T / 2\right), \\
\Phi_{10}^{\mathrm{QCD}}\left(\vec{\theta}_{1}, \vec{\theta}_{2}, \vec{\theta}_{3}\right) \equiv \ln \left(\frac{k_{\mathrm{A}_{2}}^{1}\left(x_{0}, \vec{\theta}_{1}, \vec{\theta}_{2}\right)}{k_{\mathrm{A}_{2}}^{1}\left(x_{0}, \vec{\theta}_{1}, \vec{\theta}_{3}\right)}\right), & \left(T=L, x_{0}=T / 2\right), \\
\Phi_{11}^{\mathrm{QCD}}\left(\vec{\theta}_{1}\right) & \equiv \ln \left(\frac{J_{\mathrm{A}_{1}}^{1}\left(x_{0}, \vec{\theta}_{1}, \vec{\theta}_{1}, \vec{\theta}_{1}\right)}{\sqrt{F_{1}\left(\vec{\theta}_{1}, \vec{\theta}_{1}\right) \times K_{1}^{\ell \ell}\left(\vec{\theta}_{1}, \vec{\theta}_{1}\right)}}\right),\left(T=L, x_{0}=T / 2\right) .
\end{array}
$$

We prefer to determine the normalization factor of the spatial components of the axial current through $\Phi_{11}^{\mathrm{QCD}}$ which uses a boundary-to-boundary correlation function (corresponding to a three-point function in large volume) as proposed in [20]. Alternatively, we could also use

$$
\Phi_{11}^{\prime \mathrm{QCD}}\left(\vec{\theta}_{1}\right) \equiv \ln \left(\frac{f_{\overrightarrow{\mathrm{A}}}\left(x_{0}, \vec{\theta}_{1}, \vec{\theta}_{1}\right)}{\sqrt{F_{1}\left(\vec{\theta}_{1}, \vec{\theta}_{1}\right)}}\right), \quad\left(T=L, x_{0}=T / 2\right),
$$

which is defined entirely in terms of boundary-to-bulk correlators (two-point functions). Our results at tree-level indicate that $\Phi_{11}^{\mathrm{QCD}}$ yields smaller higher-order corrections in $1 / m_{\mathrm{h}}$ (see section 4). This holds true already at the static order where spin-symmetry is exact. For this reason we will preferably adopt a three-point function also for the normalization of the temporal component of the vector current (see the definition of $\Phi_{14}^{\mathrm{QCD}}$ in appendix B and ref. [20] for a perturbative study at one-loop). The list of the additional correlators and the observables $\Phi_{12}^{\mathrm{QCD}}, \ldots, \Phi_{19}^{\mathrm{QCD}}$ needed for the matching of the temporal and spatial components of the heavy-light vector current are contained in appendix B.

The matching equations just state that the above observables are equal to the corresponding HQET counterparts. The explicit form of the HQET expansions according to eq. (2.4) will be discussed in the next subsection. Here, we like to point out that the freedom to select a specific kinematics through the $\vec{\theta}$ angles of eq. (2.15) is crucial in our choice of the matching observables. The same combination of correlation function, but with different kinematics, can provide sensitivity to different HQET parameters. For example, $\Phi_{4}^{\mathrm{QCD}}$ and $\Phi_{5}^{\mathrm{QCD}}$ differ only in their kinematics. In this way the HQET expansion of $\Phi_{4}^{\mathrm{HQET}}$ does not receive contributions from correlators with insertions of the total derivative operator $A_{0,2}$, while $\Phi_{5}^{\mathrm{QCD}}$ does. In other (slightly abusing) words, $\Phi_{4}^{\mathrm{QCD}}$ is sensitive to $c_{\mathrm{A}_{0,1}}$, while $\Phi_{5}^{\mathrm{QCD}}$ is sensitive to both $c_{\mathrm{A}_{0,1}}$ and $c_{\mathrm{A}_{0,2}}$. Similarly, $\Phi_{7}^{\mathrm{QCD}}$ and $\Phi_{8}^{\mathrm{QCD}}$ are sensitive to $c_{\mathrm{A}_{k, 1}}$ and $c_{\mathrm{A}_{k, 2}}$, while the analogous observables with flavour-dependent $\vec{\theta}$ angles, $\Phi_{9}^{\mathrm{QCD}}$ and $\Phi_{10}^{\mathrm{QCD}}$, are sensitive to the entire set of parameters $c_{\mathrm{A}_{k, 1}}, c_{\mathrm{A}_{k, 2}}, c_{\mathrm{A}_{k, 3}}$ and $c_{\mathrm{A}_{k, 4}}$. 
The HQET expansions of our observables will be written in terms of functions $\tilde{\eta}_{i}\left(\vec{\theta}_{\ell}\right)$ and $\tilde{\varphi}_{i}^{j}\left(\vec{\theta}_{\ell}, \vec{\theta}_{\mathrm{h}}\right)$, which are defined in appendix A, and we distinguish three cases:

(i) Observables depending on a single angle (e.g., for $i=1,3,6$, and 11)

$$
\Phi_{i}^{\mathrm{HQET}}\left(\vec{\theta}_{1}\right)=\tilde{\eta}_{i}\left(\vec{\theta}_{1}\right)+\left.\sum_{j} \tilde{\varphi}_{i}^{j}\left(\vec{\theta}_{1}, \vec{\theta}_{1}\right) \cdot \omega_{j} \equiv\left(\tilde{\eta}_{i}+\sum_{j} \tilde{\varphi}_{i}^{j} \cdot \omega_{j}\right)\right|_{\vec{\theta}_{\ell}=\vec{\theta}_{\mathrm{h}}=\vec{\theta}_{1}}
$$

(ii) Observables depending on two angles (e.g., for $i=2,4,7$, and 8)

$$
\begin{aligned}
\Phi_{i}^{\mathrm{HQET}}\left(\vec{\theta}_{1}, \vec{\theta}_{2}\right) & =\tilde{\eta}_{i}\left(\vec{\theta}_{1}\right)-\tilde{\eta}_{i}\left(\vec{\theta}_{2}\right)+\sum_{j}\left(\tilde{\varphi}_{i}^{j}\left(\vec{\theta}_{1}, \vec{\theta}_{1}\right)-\tilde{\varphi}_{i}^{j}\left(\vec{\theta}_{2}, \vec{\theta}_{2}\right)\right) \cdot \omega_{j} \\
& \equiv\left[\tilde{\eta}_{i}+\sum_{j} \tilde{\varphi}_{i}^{j} \cdot \omega_{j}\right]_{\vec{\theta}_{\ell}=\vec{\theta}_{\mathrm{h}}=\vec{\theta}_{2}}^{\vec{\theta}_{\ell}=\vec{\theta}_{\mathrm{h}}=\vec{\theta}_{1}}
\end{aligned}
$$

(iii) Observables depending on three angles (e.g., for $i=5,9$, and 10)

$$
\begin{aligned}
\Phi_{i}^{\mathrm{HQET}}\left(\vec{\theta}_{1}, \vec{\theta}_{2}, \vec{\theta}_{3}\right) & =\sum_{j}\left(\tilde{\varphi}_{i}^{j}\left(\vec{\theta}_{1}, \vec{\theta}_{2}\right)-\tilde{\varphi}_{i}^{j}\left(\vec{\theta}_{1}, \vec{\theta}_{3}\right)\right) \cdot \omega_{j} \\
& \equiv\left[\sum_{j} \tilde{\varphi}_{i}^{j} \cdot \omega_{j}\right]_{\vec{\theta}_{\ell}=\vec{\theta}_{1}, \vec{\theta}_{\mathrm{h}}=\vec{\theta}_{3}}^{\vec{\theta}_{\ell}=\vec{\theta}_{1}, \vec{\theta}_{\mathrm{h}}=\vec{\theta}_{2}}
\end{aligned}
$$

where any contribution from $\tilde{\eta}_{i}\left(\vec{\theta}_{\ell}\right)$, which only depends on $\vec{\theta}_{\ell}$, can be dropped because it cancels in the difference.

Observables of the form (i) are needed for the determination of HQET parameters, like $m_{\text {bare }}$ or $Z_{J}$ factors, which determine the overall (re-)normalization of physical quantities. To illustrate the physical situations exploited by the observables of the form (ii) and (iii), we should keep in mind that in the SF the boundary fields create states which in the limit of large $T$ (and spatial volume) would include complicated multi-particle states (e.g., a B-meson plus a certain number of pions, including their excited states). Their total momentum is proportional to $\vec{\theta}_{\mathrm{h}}-\vec{\theta}_{\ell}$. Thus, observables of the form (ii) probe two different states with vanishing total momentum, while in (iii) one compares two states with also different total momentum (by giving different momenta to the heavy quark).

At tree-level and without background field all correlation functions with an insertion of $O_{\text {spin }}$ vanish. In this case, none of the observables is sensitive to $\omega_{\text {spin }}$ (but it is also not needed to determine any of the other parameters). To ensure sensitivity to $\omega_{\text {spin }}$ at tree-level, one could use a different setup with a non-trivial background field, similar to the one adopted in ref. [26] for the computation of the renormalization constant of the $O_{\text {spin }}$ operator. In refs. [7, 10] it has been shown that in any case at the non-perturbative level a good sensitivity to $\omega_{\text {spin }}$ can be obtained also with vanishing background field.

\subsection{HQET parameters of the Lagrangian and of the axial current}

We describe how the system of matching equations can be solved. We use the shorthand notation introduced in the previous subsection and suppress the arguments $L$ and $a$. The explicit expressions for $\tilde{\eta}_{i}\left(\vec{\theta}_{\ell}\right)$ and $\tilde{\varphi}_{i}^{j}\left(\vec{\theta}_{\ell}, \vec{\theta}_{\mathrm{h}}\right)$ are collected in appendix A. 
The observables are constructed in such a way that the bare mass enters only in the HQET expansion of

$$
\Phi_{1}^{\mathrm{HQET}}\left(\vec{\theta}_{1}\right)=\left.\left(\tilde{\eta}_{1}+\tilde{\varphi}_{1}^{1} \cdot m_{\text {bare }}+\tilde{\varphi}_{1}^{2} \cdot \omega_{\mathrm{kin}}+\tilde{\varphi}_{1}^{3} \cdot \omega_{\mathrm{spin}}+\tilde{\varphi}_{1}^{4} \cdot c_{\mathrm{A}_{0,1}}\right)\right|_{\vec{\theta}_{\ell}=\vec{\theta}_{\mathrm{h}}=\vec{\theta}_{1}},
$$

where we note that the contribution from $A_{0,1}$ would vanish if the matching were performed on a lattice of large temporal extent $\left(\right.$ as $\tilde{\varphi}_{1}^{4}\left(\vec{\theta}_{\ell}, \vec{\theta}_{\mathrm{h}}\right) \rightarrow 0$ for $\left.T \rightarrow \infty\right)$.

In order to solve the corresponding matching equation, one first needs to determine $\omega_{\text {kin }}, \omega_{\text {spin }}$, and $c_{\mathrm{A}_{0,1}}$ from the matching equations for $\Phi_{2}, \cdots, \Phi_{4}$. First,

$$
\Phi_{2}^{\mathrm{HQET}}\left(\vec{\theta}_{1}, \vec{\theta}_{2}\right)=\left[\tilde{\eta}_{2}+\tilde{\varphi}_{2}^{2} \cdot \omega_{\mathrm{kin}}\right]_{\vec{\theta}_{\ell}=\vec{\theta}_{\mathrm{h}}=\vec{\theta}_{2}}^{\vec{\theta}_{\ell}=\vec{\theta}_{\mathrm{h}}=\vec{\theta}_{1}},
$$

and

$$
\Phi_{3}^{\mathrm{HQET}}\left(\vec{\theta}_{1}\right)=\left.\tilde{\varphi}_{3}^{3} \cdot \omega_{\mathrm{spin}}\right|_{\vec{\theta}_{\ell}=\vec{\theta}_{\mathrm{h}}=\vec{\theta}_{1}}
$$

allows determining $\omega_{\text {kin }}$ and $\omega_{\text {spin }}$, respectively. Note that in the HQET expansion of $\Phi_{2}$ and $\Phi_{3}$ we have used the spin-symmetry relations $K_{1}^{\text {stat }}=F_{1}^{\text {stat }}, K_{1}^{\text {kin }}=F_{1}^{\text {kin }}$ and $K_{1}^{\text {spin }}=-\frac{1}{3} F_{1}^{\text {spin }}$.

Due to the choice $\vec{\theta}_{\ell}=\vec{\theta}_{\mathrm{h}}$, the HQET expansion

$$
\Phi_{4}^{\mathrm{HQET}}\left(\vec{\theta}_{1}, \vec{\theta}_{2}\right)=\left[\tilde{\eta}_{4}+\tilde{\varphi}_{4}^{2} \cdot \omega_{\mathrm{kin}}+\tilde{\varphi}_{4}^{3} \cdot \omega_{\mathrm{spin}}+\tilde{\varphi}_{4}^{4} \cdot c_{\mathrm{A}_{0,1}}\right]_{\vec{\theta}_{\ell}=\vec{\theta}_{\mathrm{h}}=\vec{\theta}_{2}}^{\vec{\theta}_{\ell}=\vec{\theta}_{\mathrm{h}}=\vec{\theta}_{1}}
$$

has no contribution from $A_{0,2}$. This permits us to extract $c_{\mathrm{A}_{0,1}}$, and hence eq. (3.15) can be solved for $m_{\text {bare }}$.

On the other hand, $c_{\mathrm{A}_{0,2}}$ can be determined from matching $\Phi_{5}$, which for $\vec{\theta}_{1} \neq \vec{\theta}_{2}$ or $\vec{\theta}_{1} \neq \vec{\theta}_{3}$ has the HQET expansion

$$
\Phi_{5}^{\mathrm{HQET}}\left(\vec{\theta}_{1}, \vec{\theta}_{2}, \vec{\theta}_{3}\right)=\left[\tilde{\varphi}_{5}^{2} \cdot \omega_{\mathrm{kin}}+\tilde{\varphi}_{5}^{4} \cdot c_{\mathrm{A}_{0,1}}+\tilde{\varphi}_{5}^{5} \cdot c_{\mathrm{A}_{0,2}}\right]_{\vec{\theta}_{\ell}=\vec{\theta}_{1}, \vec{\theta}_{\mathrm{h}}=\vec{\theta}_{3}}^{\vec{\theta}_{\ell}=\vec{\theta}_{1}, \vec{\theta}_{\mathrm{l}}=\vec{\theta}_{2}} .
$$

Finally, $\ln Z_{A_{0}}^{\mathrm{HQET}}$ can be computed (independently from $\Phi_{5}$ ) from matching $\Phi_{6}$, which has the HQET expansion

$$
\Phi_{6}^{\mathrm{HQET}}\left(\vec{\theta}_{1}\right)=\left.\left(\tilde{\eta}_{6}+\tilde{\varphi}_{6}^{2} \cdot \omega_{\mathrm{kin}}+\tilde{\varphi}_{6}^{3} \cdot \omega_{\mathrm{spin}}+\tilde{\varphi}_{6}^{4} \cdot c_{\mathrm{A}_{0,1}}+\ln Z_{A_{0}}^{\mathrm{HQET}}\right)\right|_{\vec{\theta}_{\mathrm{h}}=\vec{\theta}_{\ell}=\vec{\theta}_{1}} \cdot
$$

The $\vec{\theta}$ angles in the above equations can always be taken to be isotropic, i.e., $\vec{\theta}=(\theta, \theta, \theta)$. Moreover, we can use $\vec{\theta}_{\mathrm{h}}=\vec{\theta}_{\ell}$ except in $\Phi_{5}$, because otherwise $\tilde{\varphi}_{5}^{5} \sim f_{A_{0,2}}\left(\vec{\theta}_{\ell}, \vec{\theta}_{\mathrm{h}}\right)$ vanishes and hence there would be no sensitivity to $c_{\mathrm{A}_{0,2}}$.

The extension of the system of equations to include the matching of the spatial components of the axial current preserves the block structure described in section 2. In detail, to determine $c_{\mathrm{A}_{k, 1}}$ and $c_{\mathrm{A}_{k, 2}}$, the matching equations for $\Phi_{7}$ and $\Phi_{8}$ need to be solved together. Their HQET expansion is

$$
\Phi_{7}^{\mathrm{HQET}}\left(\vec{\theta}_{1}, \vec{\theta}_{2}\right)=\left[\tilde{\eta}_{7}+\tilde{\varphi}_{7}^{2} \cdot \omega_{\mathrm{kin}}+\tilde{\varphi}_{7}^{3} \cdot \omega_{\mathrm{spin}}+\tilde{\varphi}_{7}^{7} \cdot c_{\mathrm{A}_{k, 1}}+\tilde{\varphi}_{7}^{8} \cdot c_{\mathrm{A}_{k, 2}}\right]_{\vec{\theta}_{\ell}=\vec{\theta}_{\mathrm{h}}=\vec{\theta}_{2}}^{\vec{\theta}_{\ell}=\vec{\theta}_{\mathrm{h}}=\vec{\theta}_{1}},
$$


and

$$
\Phi_{8}^{\mathrm{HQET}}\left(\vec{\theta}_{1}, \vec{\theta}_{2}\right)=\left[\tilde{\eta}_{8}+\tilde{\varphi}_{8}^{2} \cdot \omega_{\mathrm{kin}}+\tilde{\varphi}_{8}^{3} \cdot \omega_{\mathrm{spin}}+\tilde{\varphi}_{8}^{7} \cdot c_{\mathrm{A}_{k, 1}}+\tilde{\varphi}_{8}^{8} \cdot c_{\mathrm{A}_{k, 2}}\right]_{\vec{\theta}_{\ell}=\vec{\theta}_{\mathrm{h}}=\vec{\theta}_{2}}^{\vec{\theta}_{\ell}=\vec{\theta}_{\mathrm{h}}=\vec{\theta}_{1}} .
$$

Analogously, for $c_{\mathrm{A}_{k, 3}}$ and $c_{\mathrm{A}_{k, 4}}$, the matching equations for $\Phi_{9}$ and $\Phi_{10}$ have to be solved. Their HQET expansion reads

$$
\Phi_{9}^{\mathrm{HQET}}\left(\vec{\theta}_{1}, \vec{\theta}_{2}, \vec{\theta}_{3}\right)=\left[\tilde{\varphi}_{9}^{2} \cdot \omega_{\mathrm{kin}}+\tilde{\varphi}_{9}^{7} \cdot c_{\mathrm{A}_{k, 1}}+\tilde{\varphi}_{9}^{8} \cdot c_{\mathrm{A}_{k, 2}}+\tilde{\varphi}_{9}^{9} \cdot c_{\mathrm{A}_{k, 3}}+\tilde{\varphi}_{9}^{10} \cdot c_{\mathrm{A}_{k, 4}}\right]_{\vec{\theta}_{\ell}=\vec{\theta}_{1}, \vec{\theta}_{\mathrm{h}}=\vec{\theta}_{3}}^{\vec{\theta}_{\ell}=\vec{\theta}_{1}, \vec{\theta}_{\mathrm{h}}=\vec{\theta}_{2}},
$$

and

$$
\begin{aligned}
\Phi_{10}^{\mathrm{HQET}}\left(\vec{\theta}_{1}, \vec{\theta}_{2}, \vec{\theta}_{3}\right)=[ & \tilde{\varphi}_{10}^{2} \cdot \omega_{\mathrm{kin}}+\tilde{\varphi}_{10}^{7} \cdot c_{\mathrm{A}_{k, 1}}+\tilde{\varphi}_{10}^{8} \cdot c_{\mathrm{A}_{k, 2}} \\
& \left.+\tilde{\varphi}_{10}^{9} \cdot c_{\mathrm{A}_{k, 3}}+\tilde{\varphi}_{10}^{10} \cdot c_{\mathrm{A}_{k, 4}}\right]_{\vec{\theta}_{\ell}=\vec{\theta}_{1}, \vec{\theta}_{\mathrm{h}}=\vec{\theta}_{3}}^{\vec{\theta}_{\ell}=\vec{\theta}_{1}, \vec{\theta}_{\mathrm{h}}=\vec{\theta}_{2}}
\end{aligned}
$$

Independently from $\Phi_{9}$ and $\Phi_{10}, \ln Z_{\vec{A}}^{\mathrm{HQET}}$ can be extracted from the matching of

$$
\begin{aligned}
\Phi_{11}^{\mathrm{HQET}}\left(\vec{\theta}_{1}\right)=( & \tilde{\eta}_{11}+\tilde{\varphi}_{11}^{2} \cdot \omega_{\mathrm{kin}}+\tilde{\varphi}_{11}^{3} \cdot \omega_{\text {spin }} \\
& \left.+\tilde{\varphi}_{11}^{7} \cdot c_{\mathrm{A}_{k, 1}}+\tilde{\varphi}_{11}^{8} \cdot c_{\mathrm{A}_{k, 2}}+\ln Z_{\vec{A}}^{\mathrm{HQET}}\right)\left.\right|_{\vec{\theta}_{\ell}=\vec{\theta}_{\ell^{\prime}}=\vec{\theta}_{\mathrm{h}}=\vec{\theta}_{1}},
\end{aligned}
$$

which uses a three-point function. Alternatively, one can take the observable as in eq. (3.14)

$$
\begin{aligned}
\Phi_{11}^{\prime \mathrm{HQET}}\left(\vec{\theta}_{1}\right)=( & \tilde{\eta}_{11}^{\prime}+\tilde{\varphi}_{11}^{\prime 2} \cdot \omega_{\mathrm{kin}}+\tilde{\varphi}_{11}^{\prime 3} \cdot \omega_{\mathrm{spin}} \\
& \left.+\tilde{\varphi}_{11}^{\prime 7} \cdot c_{\mathrm{A}_{k, 1}}+\tilde{\varphi}_{11}^{\prime 8} \cdot c_{\mathrm{A}_{k, 2}}+\ln Z_{\vec{A}}^{\mathrm{HQET}}\right)\left.\right|_{\vec{\theta}_{\ell}=\vec{\theta}_{\mathrm{h}}=\vec{\theta}_{1}},
\end{aligned}
$$

which is defined through two-point functions only.

\section{Tree-level results for heavy-light currents at $\mathcal{O}\left(1 / m_{\mathrm{h}}\right)$}

We perform the tree-level computation using $\mathcal{O}(a)$ improved Wilson quarks. For the static action, at this order, the Eichten-Hill discretization in ref. [27] and the HYP1/2 actions in ref. [28] coincide. We numerically evaluate the tree-level expressions for the relevant correlation functions relying on the known formulae for the relativistic and static SF-propagators. Those can be found in refs. [19, 29]. In QCD we fix $z=\{4,8,12,16,20,24,28,32,64\}$ with $z=\tilde{m}_{\mathrm{q}} L$ and

$$
\tilde{m}_{\mathrm{q}}=m_{\mathrm{q}}\left(1+b_{\mathrm{m}} a m_{\mathrm{q}}\right), \quad a m_{\mathrm{q}}=\frac{1}{2}\left(\frac{1}{\kappa}-\frac{1}{\kappa_{\mathrm{c}}}\right),
$$

where $\kappa_{\mathrm{c}}$ is the critical value of the Wilson hopping-parameter and $b_{\mathrm{m}}$ is an improvement coefficient [24]. At tree-level $\kappa_{\mathrm{c}}=1 / 8$ and $b_{\mathrm{m}}=-1 / 2$. Of course, at this order other definitions of the heavy-quark mass could have been used (see, e.g., the one adopted in appendix D of ref. [7]). The one we chose is the simplest consistent with $\mathcal{O}(a)$ improvement.

At tree-level we can take the continuum limit of each individual term in the HQET expansion. We do that by a linear extrapolation in $a^{2}$ of results obtained for $128 \leq L / a \leq$ 


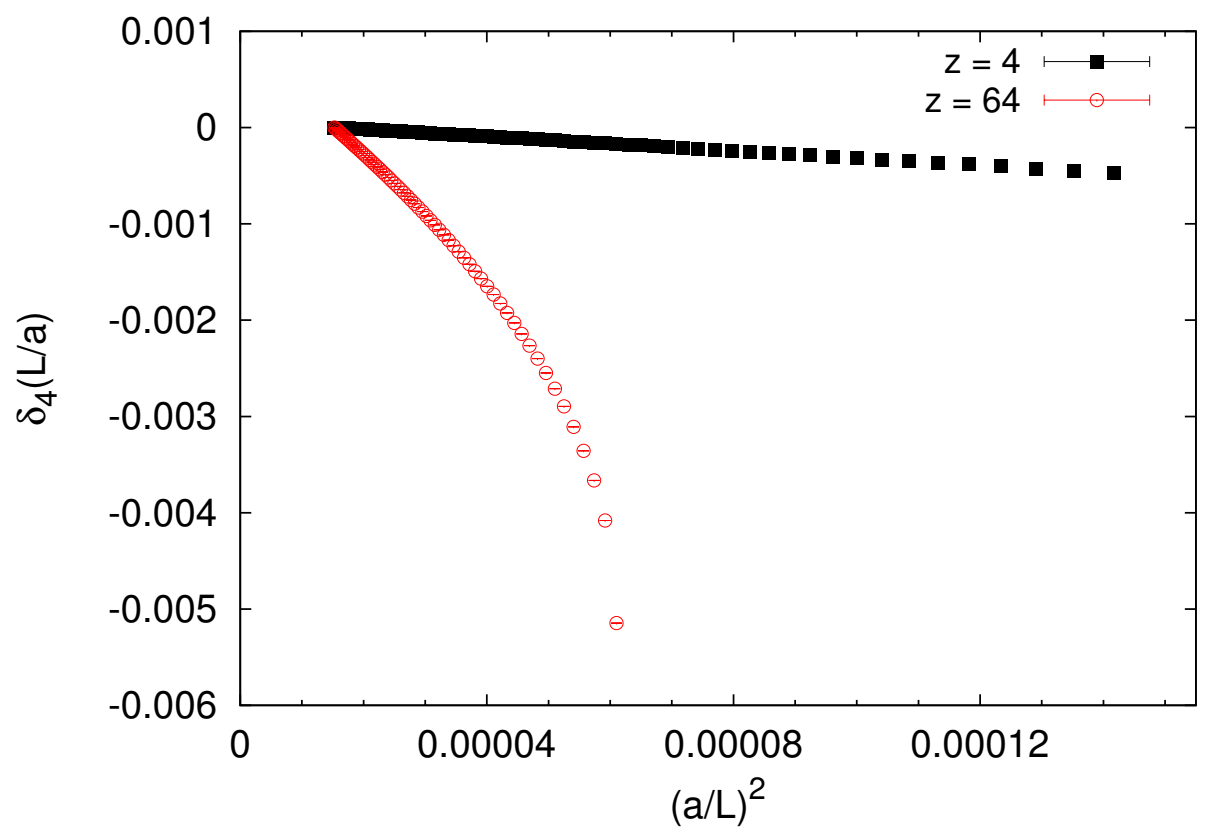

Figure 1. Tree-level results for $\delta_{4}(L / a)$ defined as the difference between $\Phi_{4}^{\mathrm{QCD}}(1,0.5)$ at a given value of $L / a$ and $\Phi_{4}^{\mathrm{QCD}}(1,0.5)$ at $L / a=256$, for $z=4$ and $z=64$.

256. We adopt the same procedure for the quantities computed in QCD at each value of $z$. These extrapolations are always well controlled, however, given the high precision of the available data, the growth of cutoff effects with $z$ is clearly visible in the QCD results; we provide an example in figure 1 . Therefore, $a^{3}$-terms as well as $a^{4}$-terms had sometimes to be included in the fits.

Once we have performed the continuum limit extrapolations for all the $\Phi_{i}^{\mathrm{QCD}}, \eta_{i}$, and $\varphi_{i}^{j}$ quantities, we solve the system of matching equations (2.4) for the unknown HQET parameters

$$
\omega_{k}=\left(\varphi^{-1}\right)_{k}^{i} \cdot\left(\Phi_{i}^{\mathrm{QCD}}-\eta_{i}\right)
$$

Due to the block structure of the matrix $\varphi$ and to the additional simplifications arising at tree-level, the parameters can be determined one after the other. For instance, making use of tree-level relations as those in appendix D of ref. [7], one finds that $\tilde{\varphi}_{11}^{2}$ (using either three- or two-point functions) and $\tilde{\varphi}_{6}^{2}$ vanish in the continuum. We also recall that all the terms proportional to $\omega_{\text {spin }}$ vanish at tree-level.

As an example we consider $\Phi_{4}^{\mathrm{QCD}}$. In order to determine $c_{\mathrm{A}_{0,1}}$ from

$$
\frac{\left(\Phi_{4}^{\mathrm{QCD}}\left(L, \tilde{m}_{\mathrm{q}}, 0\right)-\eta_{4}(L, 0)-\varphi_{4}^{2}(L, 0) \omega_{\mathrm{kin}}\right)}{\varphi_{4}^{4}(L, 0)}=c_{\mathrm{A}_{0,1}},
$$

one has to first determine other parameters, namely $\omega_{\text {kin }}$ in this case. ${ }^{1}$ Then, with $c_{\mathrm{A}_{0,1}}$, one can determine $m_{\text {bare }}, c_{\mathrm{A}_{0,2}}$, and $\ln Z_{A_{0}}^{\mathrm{HQET}}$, etc.

\footnotetext{
${ }^{1} \mathrm{~A}$ term $-\varphi_{4}^{3}(L, 0) \omega_{\text {spin }}$ in the numerator on the l.h.s. of eq. (4.3) has been dropped as it vanishes at tree-level.
} 
In order to investigate the size of higher-order contributions in $1 / m_{\mathrm{h}}$, we multiply each parameter with appropriate factors of $m_{\mathrm{h}} \equiv \tilde{m}_{\mathrm{q}}$ or $L$. Then, for $z \equiv m_{\mathrm{h}} \cdot L \rightarrow \infty$, these combinations approach the known classical values, for example

$$
\begin{aligned}
c_{\mathrm{A}_{0,1}} \cdot m_{\mathrm{h}} & =-\frac{1}{2}+\mathcal{O}(1 / z), \\
\ln Z_{A_{0}}^{\mathrm{HQET}} & =0+\mathcal{O}(1 / z), \\
m_{\text {bare }}-m_{\mathrm{h}} & =0+\mathcal{O}(1 / z) .
\end{aligned}
$$

Note that in the case of $\omega_{1} \equiv m_{\text {bare }}$ both, the term of order $m_{\mathrm{h}}$ and the term of order 1 (which vanishes at tree level), are already fixed by matching in the static approximation. Any $\mathcal{O}(1 / z)$ deviations from the above behaviour are then a sign of the $1 / m_{\mathrm{h}}$-corrections neglected in the static theory.

If all $1 / m_{\mathrm{h}}$-terms are included in HQET and in the matching with $\mathrm{QCD}$, as done throughout this paper, then also the $\mathcal{O}(1 / z)$-corrections to the parameters are fixed. The deviation from a linear $1 / z$-dependence is then a sign of higher-order corrections neglected in the effective theory. These corrections also give rise to a non-vanishing dependence of the parameters on the specific $\vec{\theta}$ angles in the choice of the observables used in the matching.

When solving the system of matching equations by backward substitution, the higherorder corrections in $1 / z$ enter through the non-linear $1 / z$-dependence of the already determined parameters as well as through the higher-order $1 / z$-dependence of the QCD observable itself. To disentangle these two sources we solve each equation in two ways: either we directly use the results of the previously determined parameters at the same value of $z$ and for the choice of $\vec{\theta}$ angles, which gave the smallest higher-order corrections in $1 / z$, or we use their classical values, e.g., $-1 /\left(2 m_{\mathrm{h}}\right)$ for $\omega_{\text {kin }}$ in the equation for $c_{\mathrm{A}_{0,1}}$. The $1 / z$-dependence of the parameters obtained in both ways is shown in the figures $2-6$. The values obtained in the latter way are labeled with a superscript "EX", indicating that in this case the $1 / z$-corrections are exclusively due to the QCD observable and not inherited from previously determined parameters.

The parameter $\omega_{\text {kin }}$ enters in the matching equations for many of the other parameters. From figure 2 we see that $\omega_{\text {kin }}$ has large $1 / z$-corrections, although they are to a very good approximation linear in $1 / z$ and $\vec{\theta}$-independent. Propagation of these large effects into other parameters, where $\omega_{\text {kin }}$ enters in the matching equation, is then suppressed in the "EX" setup.

Although it is rather difficult to single out a unique choice for the $\vec{\theta}$ angles producing the smallest higher-order corrections across all parameters, we collect in table 1 those values which produced the smallest higher-order corrections for each parameter (and for $z \geq 10$ ). The selection was performed without the "EX" option in order to stay in a situation which is closer to the one encountered when performing non-perturbative matching. Of course, our choice has been made only from a small set of $\vec{\theta}$ angles (in a rather large range), thus it can only give an rough indication of the optimal values.

From the lower panels of figures $2-6$, we note that the choice $\vec{\theta}_{1}=0$ is clearly the best for $m_{\text {bare }}, \ln Z_{A_{0}}, \ln Z_{\overrightarrow{\mathrm{A}}}$, $\ln Z_{V_{0}}$ and $\ln Z_{\overrightarrow{\mathrm{V}}}$. These are the parameters determined from observables which depend only on a single angle $\vec{\theta}_{1}$ (corresponding to case (i) of section 3.2). 


\begin{tabular}{|lccc|}
\hline parameter & $\vec{\theta}_{1}$ & $\vec{\theta}_{2}$ & $\vec{\theta}_{3}$ \\
\hline$m_{\text {bare }}$ & 0.0 & - & - \\
$\omega_{\text {kin }}$ & $0.0-0.5$ & $0.5-1.0(*)$ & - \\
\hline$c_{\mathrm{A}_{0,1}}$ & 0.0 & 0.5 & - \\
$c_{\mathrm{A}_{0,2}}$ & 0.5 & 0.0 & 1.0 \\
$\ln Z_{A_{0}}$ & 0.0 & - & - \\
$c_{\mathrm{A}_{k, 1}}$ & $0.5-1.5$ & $0.5-1.0(*)$ & - \\
$c_{\mathrm{A}_{k, 2}}$ & $0.5-1.5$ & $0.5-1.5(*)$ & - \\
$c_{\mathrm{A}_{k, 3}}$ & 0.5 & 1.5 & 1.0 \\
$c_{\mathrm{A}_{k, 4}}$ & 0.5 & 1.0 & 0.0 \\
$\ln Z_{A_{k}}$ & 0.0 & - & - \\
\hline$c_{\mathrm{V}_{0,1}}$ & $0.5-1.5$ & $0.5-1.5(*)$ & - \\
$c_{\mathrm{V}_{0,2}}$ & 0.5 & 0.5 & 1.0 \\
$\ln Z_{V_{0}}$ & 0.0 & - & - \\
$c_{\mathrm{V}_{k, 1}}$ & $0.0,0.5,0.5$ & $0.0,1.0,1.0$ & - \\
$c_{\mathrm{V}_{k, 2}}$ & 1.0 & 1.5 & - \\
$c_{\mathrm{V}_{k, 3}}$ & $0.0,1.5,1.5$ & $0.0,0.5,0.5$ & $0.0,1.0,1.0$ \\
$c_{\mathrm{V}_{k, 4}}$ & 1.5 & 0.5 & 1.0 \\
$\ln Z_{V_{k}}$ & 0.0 & - & - \\
\hline
\end{tabular}

Table 1. Our preferred choice of $\vec{\theta}$ angles to reduce higher-order corrections in the parameters. The $(*)$ indicates that $\vec{\theta}_{1} \neq \vec{\theta}_{2}$ is required. The shorthand $\vec{\theta}=t$ is used for $\vec{\theta}=(t, t, t)$.

At tree-level the dependence of these matching equations on any other parameter vanishes for $\vec{\theta}_{1}=0$. As a consequence, these parameters do not depend on whether one uses the "EX" setup or not, and the higher-order corrections seem to vanish. On the other hand, already at $\vec{\theta}=\overrightarrow{1}$ the corrections are very significant, especially in the static approximation.

For the parameters determined from observables which depend on two angles (corresponding to case (ii) of section 3.2), like $c_{\mathrm{A}_{0,1}}, c_{\mathrm{A}_{k, 1}}, c_{\mathrm{A}_{k, 2}}, c_{\mathrm{V}_{0,1}}, c_{\mathrm{V}_{k, 1}}$, and $c_{\mathrm{V}_{k, 2}}$, the dependence on the $\vec{\theta}$ angles is moderate, see upper panels of figures $3-6$. Smaller values of $\left(\vec{\theta}_{1}, \vec{\theta}_{2}\right)$ often yield smaller or less non-linear $1 / z$-corrections. Moreover, some of the higher-order $1 / z$-dependence is inherited from the other parameters because it tends to be smaller in the "EX" setup.

The parameters determined from observables which depend on three angles typically can depend significantly on the choice of these angles, and the effect of higher-order corrections in $1 / z$ is sizable. These effects do not decrease strongly (if at all) in the "EX" case. Therefore they are a genuine property of these observables rather than inherited from other parameters. 
Finally, in figure 7 we compare the two determinations of $\ln Z_{\mathrm{V}_{0}}^{\mathrm{HQET}}$, using either twoor three-point functions. Because of the significantly flatter $1 / z$-dependence, the latter is clearly preferable. The analogous conclusion is found for $\ln Z_{\overrightarrow{\mathrm{A}}}^{\mathrm{HQET}}$.

For the parameters that enter in the HQET expansion of $A_{0}$ and $V_{k}$, the higher-order corrections seem to be inherited to a large extent from $\omega_{\text {kin }}$. Since $A_{0}$ and $V_{k}$ are the current components relevant for the computations of the $\mathrm{B}_{(\mathrm{s})}$-meson decay constant and the form factors of semileptonic decays to light mesons, it would be important to find a way to improve the corresponding matching conditions and the one for $\omega_{\text {kin }}$. We describe a possible modification of all the observables to be used in the non-perturbative matching, which exploits the tree-level results in order to reduce the higher-order effects in $1 / z$. We consider eq. (2.4) at tree-level and replace all the parameters $\omega_{i}$ by their known classical values $\omega_{i}^{\text {cl }}$. This allows us to define a set of coefficients $\delta_{\Phi_{i}}^{\text {tree }}(L, M)$ through

$$
\Phi_{i}^{\mathrm{QCD}, \text { tree }}(L, M, 0)=\left(\eta_{i}^{\text {tree }}(L, 0)+\varphi_{i}^{j, \text { tree }}(L, 0) \omega_{j}^{\mathrm{cl}}(M)\right) \times\left(1+\delta_{\Phi_{i}}^{\text {tree }}(L, M)\right) .
$$

Clearly, we are free to modify the matching condition by higher-order terms, e.g., $\mathcal{O}\left(1 / z^{2}\right)$. In particular, we may use $\Phi_{i}^{\mathrm{QCD}}(L, M, 0) /\left(1+\delta_{\Phi_{i}}^{\text {tree }}(L, M)\right)$ in the non-perturbative matching. In this way, higher-order effects in $1 / z$ are completely removed at tree-level. The coefficients $\delta_{\Phi_{i}}^{\text {tree }}(L, M)$ are a rather simple byproduct of the results presented here and will be published together with the non-perturbative study.

\section{Conclusions}

We have presented a full set of matching conditions between HQET at $\mathcal{O}\left(1 / m_{\mathrm{h}}\right)$ and QCD for all the components of the axial and vector currents. This is the first time such an extended matching problem is formulated and solved on the lattice. The matrix of the linear system of equations to be solved in order to determine the 19 HQET parameters has a simple block structure. Therefore, once the 3 parameters of the Lagrangian have been computed, the system can then be solved independently for the parameters of each of the additional currents. The matching between QCD and HQET is performed with suitable observables in a finite volume with SF boundary conditions and exploits different kinematic situations by choosing appropriate twist angles $\vec{\theta}$. We have evaluated and solved the resulting matching equations at tree-level of perturbation theory and in the continuum limit. Thus, we have tested the feasibility of this strategy and demonstrated the sensitivity to all parameters (except for $\omega_{\text {spin }}$, which does not enter at tree-level with vanishing background field).

In our tree-level computation we have also analysed the size of higher-order corrections (in $1 / m_{\mathrm{h}}$ ), which show up through the dependence of the parameters on higher powers of $1 / z$ and on the $\vec{\theta}$ angles. The block structure of the system of equations, which is further simplified at tree-level, allowed us to independently investigate subsets of the 19 parameters. In this way, we have identified a good kinematical setup, i.e., a choice of the $\vec{\theta}$ angles, to be adopted in the non-perturbative matching. In view of a numerical implementation of that, the choice should also aim at minimizing the number of quark propagators to be computed or stored, i.e., at minimizing the number of combinations of $\vec{\theta}$ angles. 

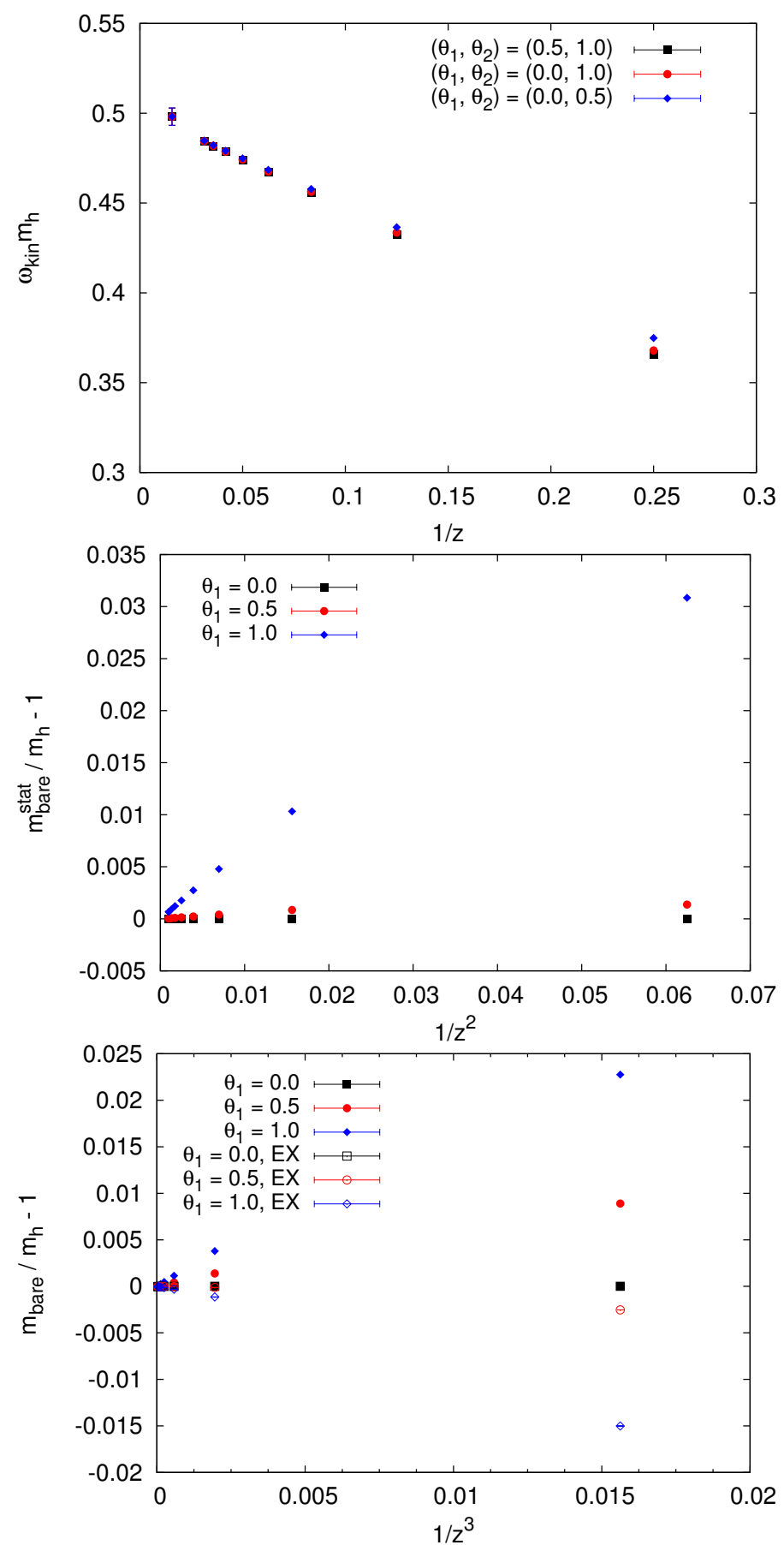

Figure 2. Tree-level continuum results for the parameters in $\mathscr{L}^{\mathrm{HQET}}$.

Remarkably, we find that in most of the cases the linear behaviour sets in already for $z \geq 8$, with slopes that are of $\mathcal{O}(1)$. This is quite satisfactory, as it means that for the non-perturbative setup chosen by the ALPHA Collaboration [10], with $L \approx 0.5 \mathrm{fm}$ and $z$ around 13 at the b-quark mass, higher-order corrections are suppressed by a factor of about 10 . 

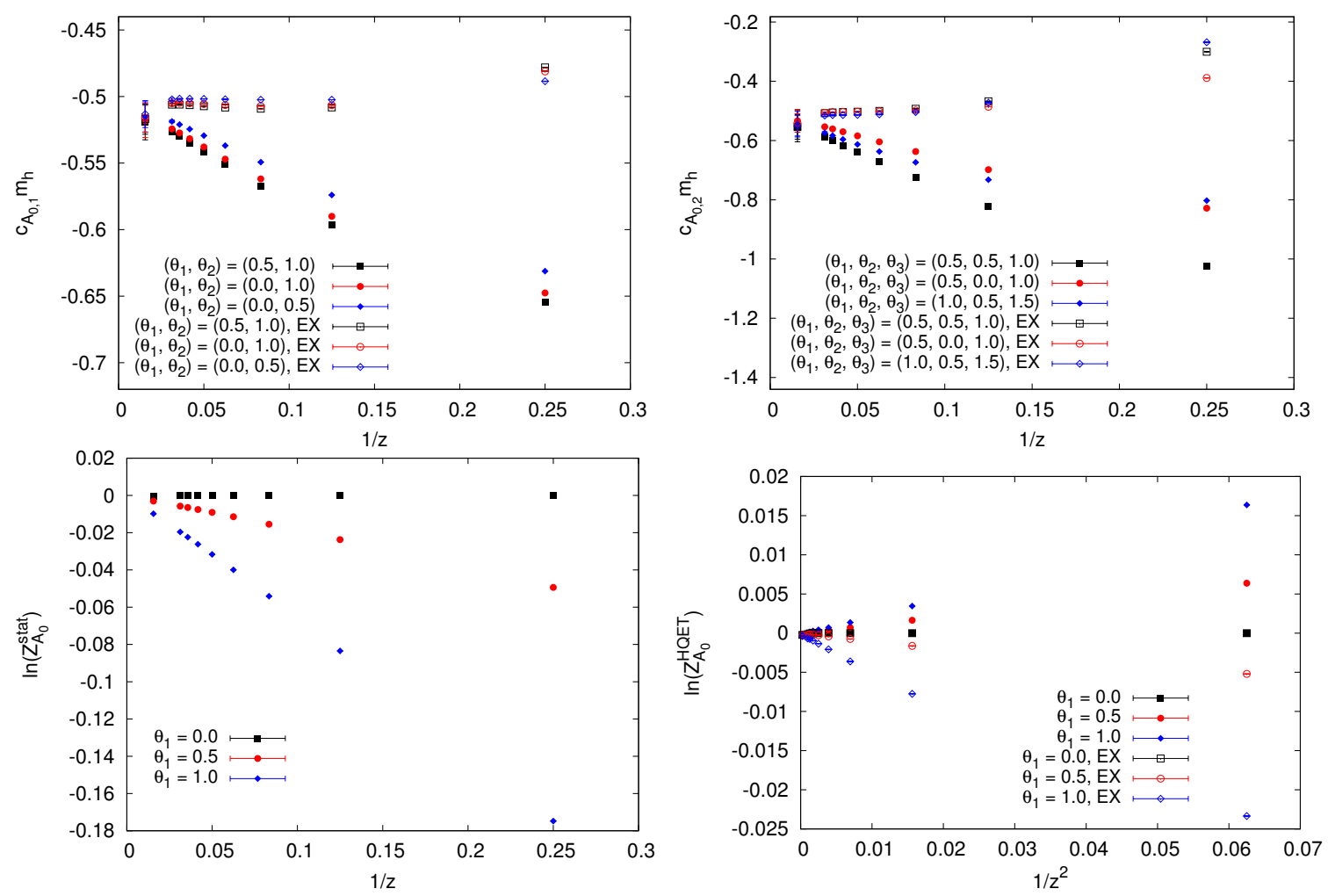

Figure 3. Tree-level continuum results for the parameters of the temporal component of the axial current.

Solving the matching problem is a necessary step towards a precise computation, within the framework of lattice HQET, of several phenomenologically relevant form factors describing semileptonic decays of $\mathrm{B}_{(\mathrm{s})}$-mesons. The matching strategy presented here is currently investigated in further detail at the one-loop order in perturbation theory [30] (see also ref. [31] for partial results), and shall then be applied non-perturbatively in numerical simulations in the near future and for the complete set of 19 matching coefficients. We use Wilson fermions but, since the matching conditions are espressed in terms of renormalized quantities, any regularization could in principle be used. Past experience shows that very fine lattice spacings and good precision could be reached with Wilson fermions [7, 10].

\section{Acknowledgments}

We thank Rainer Sommer and Piotr Korcyl for many helpful discussions and comments, and for carefully reading the manuscript. This work was partially supported by the Spanish Minister of Education and Science, project RyC-2011-08557 (M. D. M.), by the grant HE 4517/3-1 of the Deutsche Forschungsgemeinschaft (J. H.) and by the REA of the European Union under Grant Agreement number PITN-GA-2009-238353, ITN STRONGnet (D. H.). 

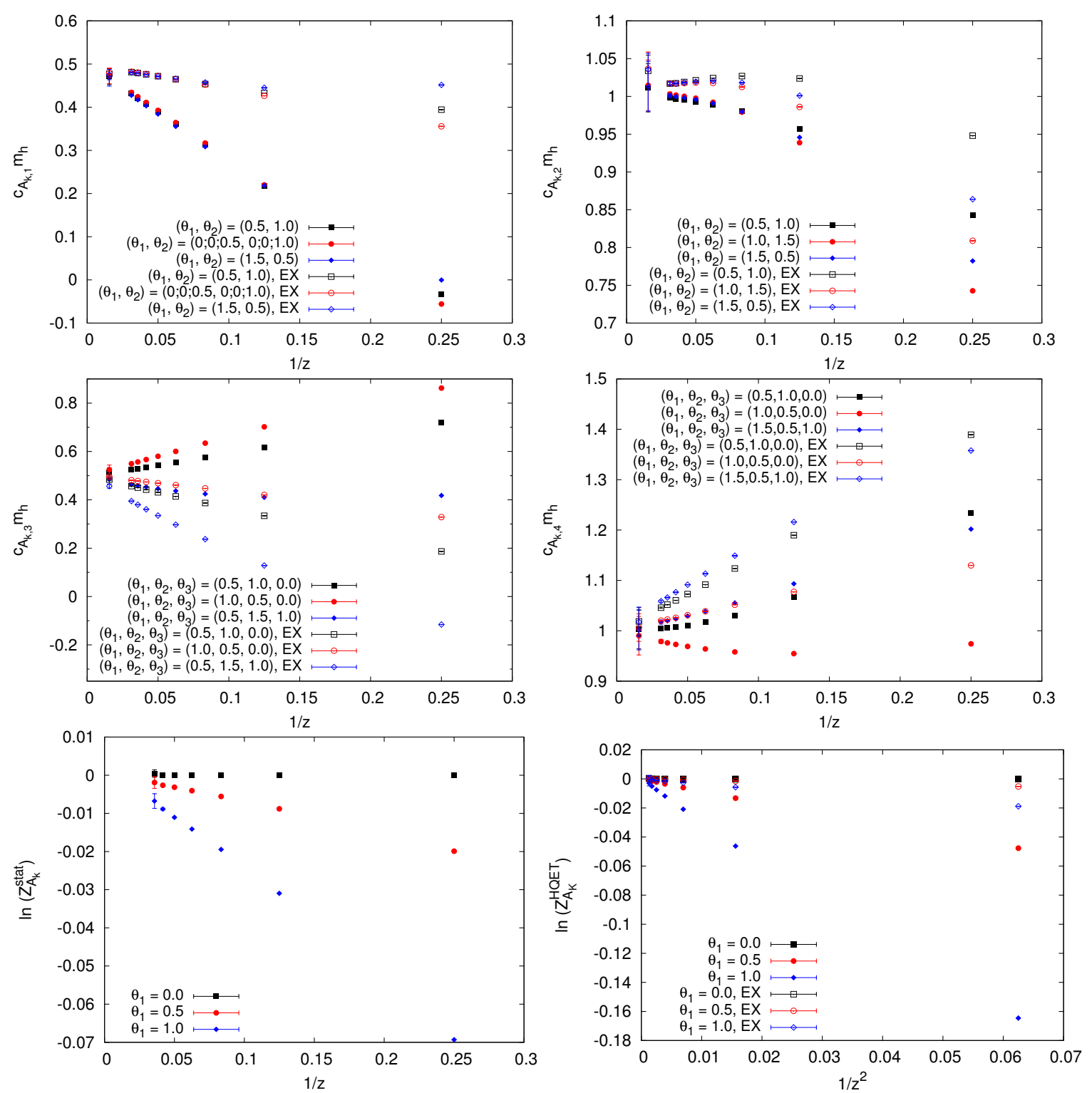

Figure 4. Tree-level continuum results for the parameters of the spatial components of the axial current.

\section{A Explict form of the matching matrix for $\mathscr{L}^{\mathrm{HQET}}, \boldsymbol{A}_{0}$ and $\boldsymbol{A}_{k}$}

In this appendix we give the explicit definition of the various $\tilde{\eta}_{i}\left(\vec{\theta}_{\ell}\right)$ and $\tilde{\varphi}_{i}^{j}\left(\vec{\theta}_{\mathrm{h}}, \vec{\theta}_{\ell}\right)$ used in the HQET expansions in section 3.

$\Phi_{1}^{\mathrm{HQET}}\left(\vec{\theta}_{1}\right)$ : The quantities in the HQET expansion, eq. (3.15), are

$$
\begin{aligned}
\tilde{\eta}_{1}\left(\vec{\theta}_{\ell}\right) & \equiv-\left.L \tilde{\partial}_{0} \ln \left(-f_{\mathrm{A}_{0}}^{\mathrm{stat}}\left(x_{0}, \vec{\theta}_{\ell}\right)\right)\right|_{x_{0}=T / 2} \\
\tilde{\varphi}_{1}^{1} & \equiv L
\end{aligned}
$$



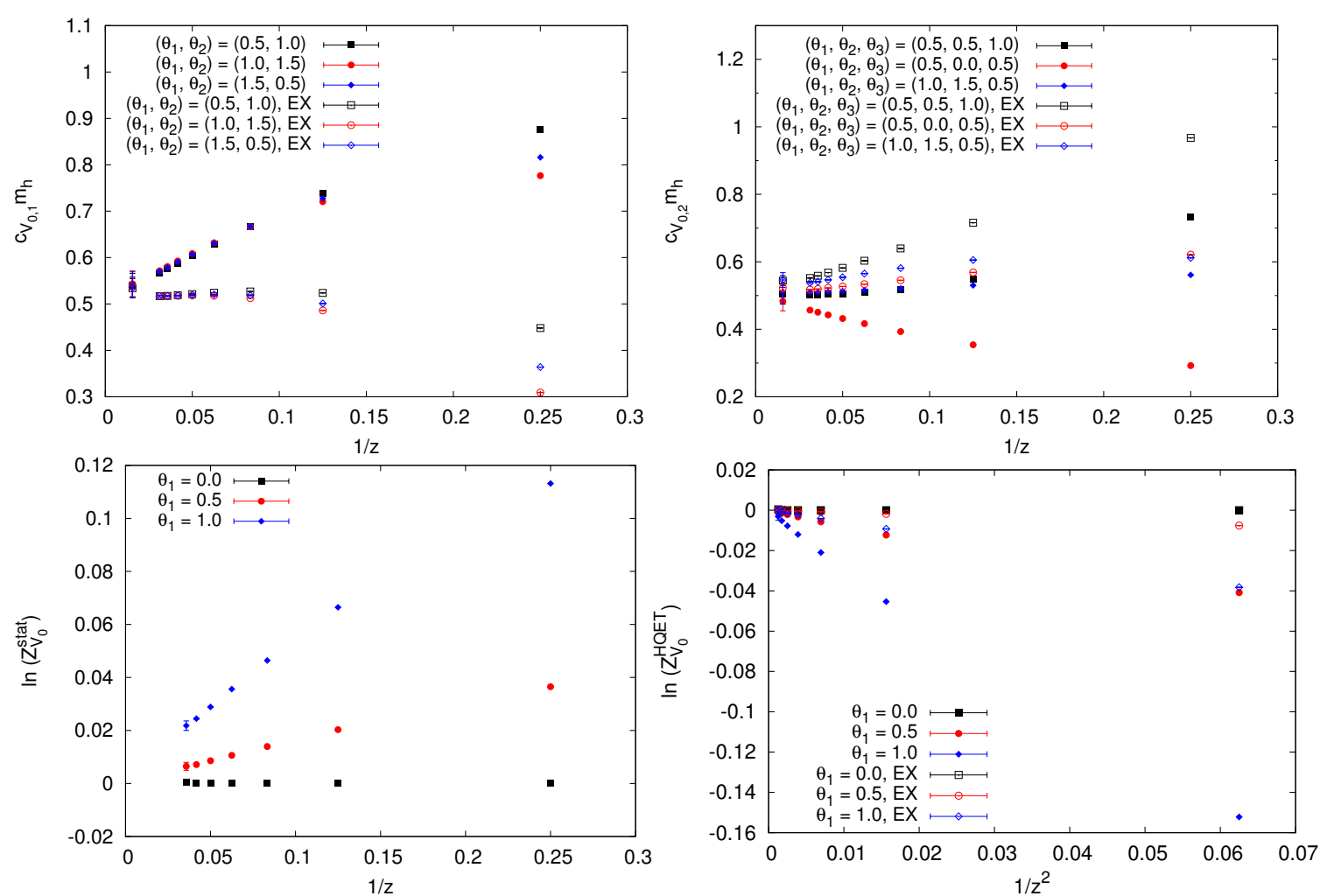

Figure 5. Tree-level continuum results for the parameters of the temporal component of the vector current.

$$
\begin{gathered}
\tilde{\varphi}_{1}^{2}\left(\vec{\theta}_{\ell}, \vec{\theta}_{\mathrm{h}}\right) \equiv-\left.L \tilde{\partial}_{0}\left(\frac{f_{\mathrm{A}_{0}}^{\mathrm{kin}}\left(x_{0}, \vec{\theta}_{\ell}, \vec{\theta}_{\mathrm{h}}\right)}{f_{\mathrm{A}_{0}}^{\mathrm{stat}}\left(x_{0}, \vec{\theta}_{\ell}\right)}\right)\right|_{x_{0}=T / 2}, \\
\tilde{\varphi}_{1}^{3}\left(\vec{\theta}_{\ell}\right) \equiv-\left.L \tilde{\partial}_{0}\left(\frac{f_{\mathrm{A}_{0}}^{\mathrm{spin}}\left(x_{0}, \vec{\theta}_{\ell}\right)}{f_{\mathrm{A}_{0}}^{\text {stat }}\left(x_{0}, \vec{\theta}_{\ell}\right)}\right)\right|_{x_{0}=T / 2}, \\
\tilde{\varphi}_{1}^{4}\left(\vec{\theta}_{\ell}, \vec{\theta}_{\mathrm{h}}\right) \equiv-\left.L \tilde{\partial}_{0}\left(\frac{f_{\mathrm{A}_{0,1}}\left(x_{0}, \vec{\theta}_{\ell}, \vec{\theta}_{\mathrm{h}}\right)}{f_{\mathrm{A}_{0}}^{\text {stat }}\left(x_{0}, \vec{\theta}_{\ell}\right)}\right)\right|_{x_{0}=T / 2} .
\end{gathered}
$$

$\Phi_{2}^{\mathrm{HQET}}\left(\vec{\theta}_{1}, \vec{\theta}_{2}\right)$ : the way correlators enter in the HQET expansion, eq. (3.16), is

$$
\begin{array}{r}
\tilde{\eta}_{2}\left(\vec{\theta}_{\ell}\right) \equiv \ln F_{1}^{\text {stat }}\left(\vec{\theta}_{\ell}\right), \\
\tilde{\varphi}_{2}^{2}\left(\vec{\theta}_{\ell}, \vec{\theta}_{\mathrm{h}}\right) \equiv \frac{F_{1}^{\mathrm{kin}}\left(\vec{\theta}_{\ell}, \vec{\theta}_{\mathrm{h}}\right)}{F_{1}^{\mathrm{stat}}\left(\vec{\theta}_{\ell}\right)} .
\end{array}
$$

$\Phi_{3}^{\mathrm{HQET}}\left(\vec{\theta}_{1}\right)$ : the way correlators enter in the HQET expansion, eq. (3.17), is

$$
\tilde{\varphi}_{3}^{3}\left(\vec{\theta}_{\ell}\right) \equiv \frac{F_{1}^{\text {spin }}\left(\vec{\theta}_{\ell}\right)}{F_{1}^{\text {stat }}\left(\vec{\theta}_{\ell}\right)} .
$$



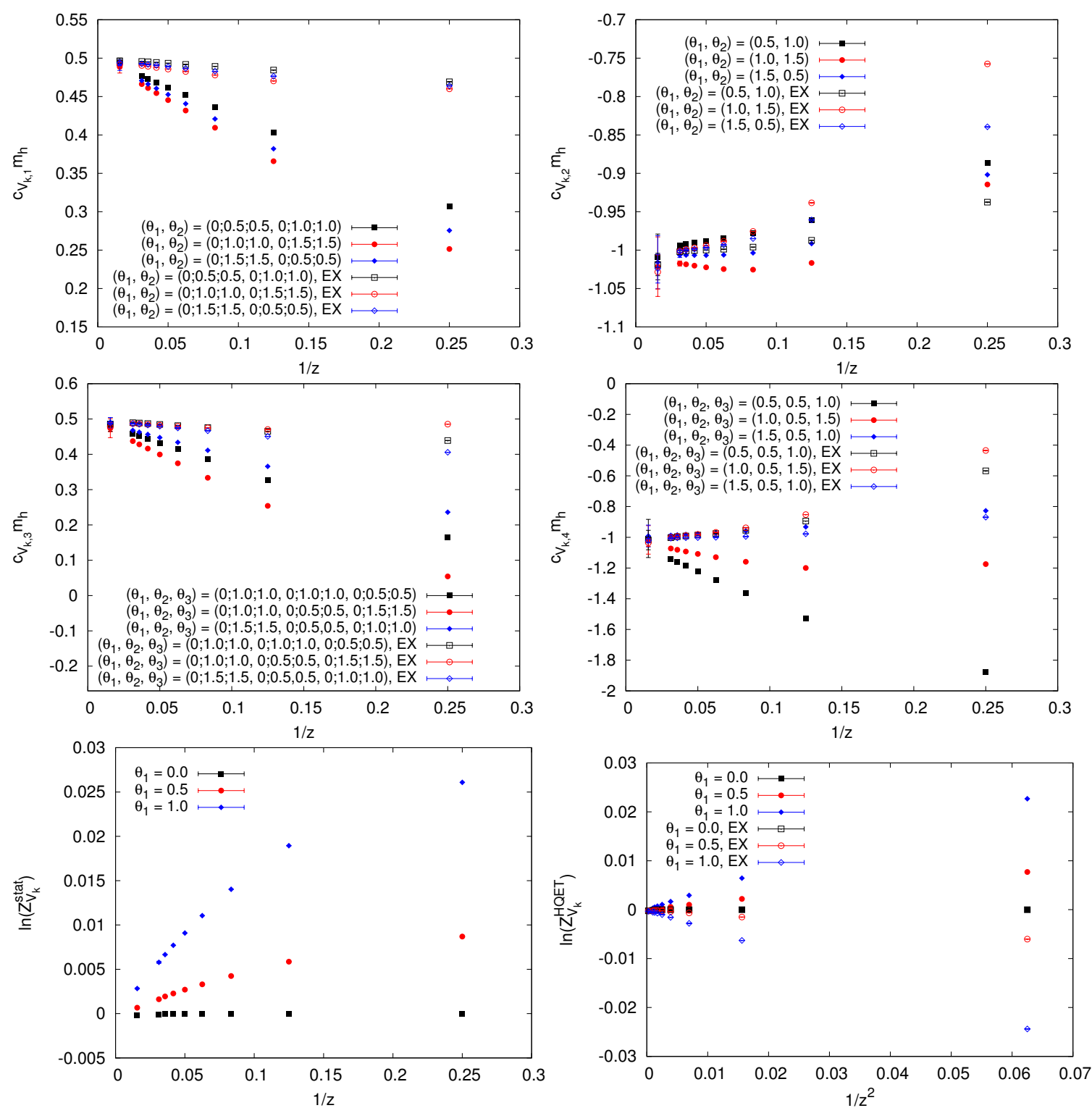

Figure 6. Tree-level continuum results for the parameters of the spatial component of the vector current.

$\Phi_{4}^{\mathrm{HQET}}\left(\vec{\theta}_{1}, \vec{\theta}_{2}\right)$ : the way correlators enter in the HQET expansion, eq. (3.18), is

$$
\begin{gathered}
\tilde{\eta}_{4}\left(\vec{\theta}_{\ell}\right) \equiv \ln f_{\mathrm{A}_{0}}^{\mathrm{stat}}\left(T / 2, \vec{\theta}_{\ell}\right), \\
\tilde{\varphi}_{4}^{2}\left(\vec{\theta}_{\ell}, \vec{\theta}_{\mathrm{h}}\right) \equiv \frac{f_{\mathrm{A}_{0}}^{\mathrm{kin}}\left(T / 2, \vec{\theta}_{\ell}, \vec{\theta}_{\mathrm{h}}\right)}{f_{\mathrm{A}_{0}}^{\mathrm{stat}}\left(T / 2, \vec{\theta}_{\ell}\right)}, \\
\tilde{\varphi}_{4}^{3}\left(\vec{\theta}_{\ell}\right) \equiv \frac{f_{\mathrm{A}_{0}}^{\mathrm{spin}}\left(T / 2, \vec{\theta}_{\ell}\right)}{f_{\mathrm{A}_{0}}^{\mathrm{stat}}\left(T / 2, \vec{\theta}_{\ell}\right)},
\end{gathered}
$$



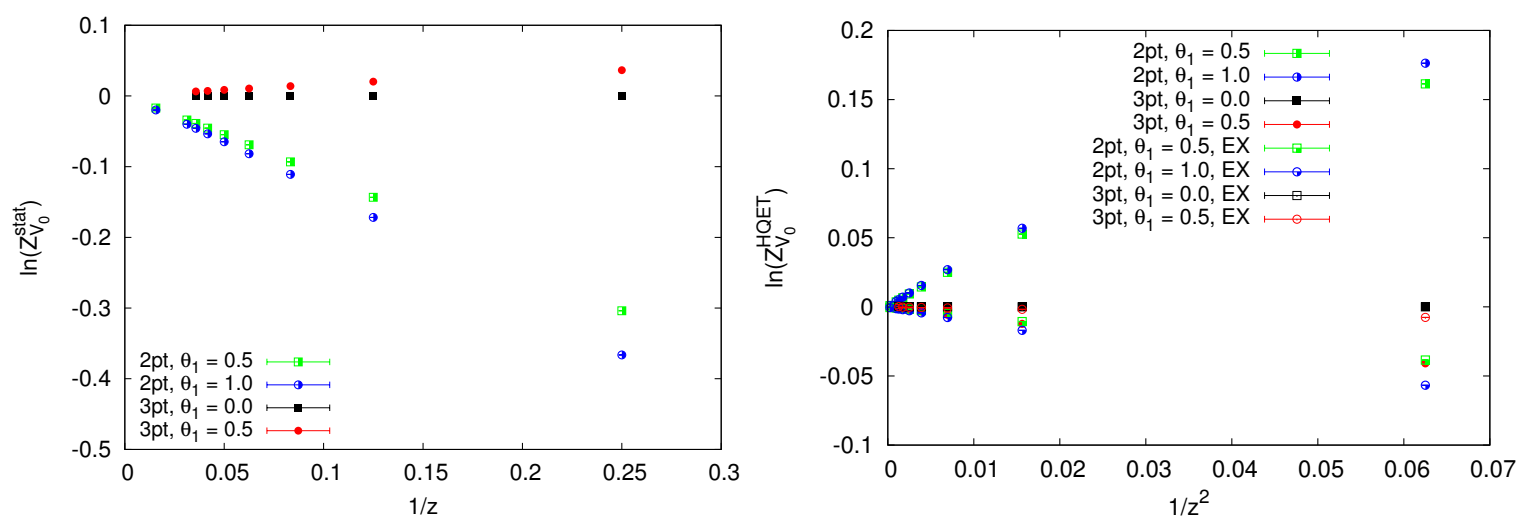

Figure 7. Comparison of tree-level continuum results for the renormalization constant of the temporal component of the vector current using either two-point correlation functions only (2pt), see eq. (B.6), or also three-point functions (3pt), as in our standard definition of $\Phi_{14}^{\mathrm{QCD}}\left(\vec{\theta}_{1}\right)$.

$$
\tilde{\varphi}_{4}^{4}\left(\vec{\theta}_{\ell}, \vec{\theta}_{\mathrm{h}}\right) \equiv \frac{f_{\mathrm{A}_{0,1}}\left(T / 2, \vec{\theta}_{\ell}, \vec{\theta}_{\mathrm{h}}\right)}{f_{\mathrm{A}_{0}}^{\text {stat }}\left(T / 2, \vec{\theta}_{\ell}\right)}
$$

$\Phi_{5}^{\mathrm{HQET}}\left(\vec{\theta}_{1}, \vec{\theta}_{2}, \vec{\theta}_{3}\right)$ : the way correlators enter in the HQET expansion, eq. (3.19), is

$$
\begin{aligned}
& \tilde{\varphi}_{5}^{2}\left(\vec{\theta}_{\ell}, \vec{\theta}_{\mathrm{h}}\right) \equiv \tilde{\varphi}_{4}^{2}\left(\vec{\theta}_{\ell}, \vec{\theta}_{\mathrm{h}}\right), \\
& \tilde{\varphi}_{5}^{4}\left(\vec{\theta}_{\ell}, \vec{\theta}_{\mathrm{h}}\right) \equiv \tilde{\varphi}_{4}^{4}\left(\vec{\theta}_{\ell}, \vec{\theta}_{\mathrm{h}}\right), \\
& \tilde{\varphi}_{5}^{5}\left(\vec{\theta}_{\ell}, \vec{\theta}_{\mathrm{h}}\right) \equiv \frac{f_{\mathrm{A}_{0,2}}\left(T / 2, \vec{\theta}_{\ell}, \vec{\theta}_{\mathrm{h}}\right)}{f_{\mathrm{A}_{0}}^{\text {stat }}\left(T / 2, \vec{\theta}_{\ell}\right)}
\end{aligned}
$$

$\Phi_{6}^{\mathrm{HQET}}\left(\vec{\theta}_{1}\right)$ : the way correlators enter in the HQET expansion, eq. (3.20), is

$$
\begin{aligned}
& \tilde{\eta}_{6}\left(\vec{\theta}_{\ell}\right) \equiv \ln \left(\frac{-f_{\mathrm{A}_{0}}^{\mathrm{stat}}\left(T / 2, \vec{\theta}_{\ell}\right)}{\sqrt{F_{1}^{\mathrm{stat}}\left(\vec{\theta}_{\ell}\right)}}\right), \\
& \tilde{\varphi}_{6}^{2}\left(\vec{\theta}_{\ell}, \vec{\theta}_{\mathrm{h}}\right) \equiv \frac{f_{\mathrm{A}_{0}}^{\mathrm{kin}}\left(T / 2, \vec{\theta}_{\ell}, \vec{\theta}_{\mathrm{h}}\right)}{f_{\mathrm{A}_{0}}^{\text {stat }}\left(T / 2, \vec{\theta}_{\ell}\right)}-\frac{1}{2} \frac{F_{1}^{\mathrm{kin}}\left(\vec{\theta}_{\ell}, \vec{\theta}_{\mathrm{h}}\right)}{F_{1}^{\mathrm{stat}}\left(\vec{\theta}_{\ell}\right)}, \\
& \tilde{\varphi}_{6}^{3}\left(\vec{\theta}_{\ell}\right) \equiv \frac{f_{\mathrm{A}_{0}}^{\mathrm{spin}}\left(T / 2, \vec{\theta}_{\ell}\right)}{f_{\mathrm{A}_{0}}^{\text {stat }}\left(T / 2, \vec{\theta}_{\ell}\right)}-\frac{1}{2} \frac{F_{1}^{\mathrm{spin}}\left(\vec{\theta}_{\ell}\right)}{F_{1}^{\text {stat }}\left(\vec{\theta}_{\ell}\right)}, \\
& \tilde{\varphi}_{6}^{4}\left(\vec{\theta}_{\ell}, \vec{\theta}_{\mathrm{h}}\right) \equiv \tilde{\varphi}_{4}^{4}\left(\vec{\theta}_{\ell}, \vec{\theta}_{\mathrm{h}}\right) .
\end{aligned}
$$

After the temporal component, we now turn our attention to the spatial components of the axial current.

For $\Phi_{7}^{\mathrm{HQET}}\left(\vec{\theta}_{1}, \vec{\theta}_{2}\right)$ in eq. $(3.21)$ :

$$
\tilde{\eta}_{7}\left(\vec{\theta}_{\ell}\right) \equiv \ln f_{\overrightarrow{\mathrm{A}}}^{\text {stat }}\left(T / 2, \vec{\theta}_{\ell}\right),
$$




$$
\begin{gathered}
\tilde{\varphi}_{7}^{2}\left(\vec{\theta}_{\ell}, \vec{\theta}_{\mathrm{h}}\right) \equiv \frac{f_{\mathrm{A}}^{\text {kin }}\left(T / 2, \vec{\theta}_{\ell}, \vec{\theta}_{\mathrm{h}}\right)}{f_{\mathrm{A}}^{\text {stat }}\left(T / 2, \vec{\theta}_{\ell}\right)}, \\
\tilde{\varphi}_{7}^{3}\left(\vec{\theta}_{\ell}\right) \equiv \frac{f_{\mathrm{A}}^{\text {spin }}\left(T / 2, \vec{\theta}_{\ell}\right)}{f_{\hat{\mathrm{A}}}^{\text {stat }}\left(T / 2, \vec{\theta}_{\ell}\right)}, \\
\tilde{\varphi}_{7}^{(}\left(\vec{\theta}_{\ell}, \vec{\theta}_{\mathrm{h}}\right) \equiv \sum_{k} \frac{f_{\mathrm{A}_{k, 1}}\left(T / 2, \vec{\theta}_{\ell}, \vec{\theta}_{\mathrm{h}}\right)}{f_{\overrightarrow{\mathrm{A}}}^{\text {stat }}\left(T / 2, \vec{\theta}_{\ell}\right)}, \\
\tilde{\varphi}_{7}^{8}\left(\vec{\theta}_{\ell}, \vec{\theta}_{\mathrm{h}}\right) \equiv \sum_{k} \frac{f_{\mathrm{A}_{k, 2}}\left(T / 2, \vec{\theta}_{\ell}, \vec{\theta}_{\mathrm{h}}\right)}{f_{\overrightarrow{\mathrm{A}}}^{\text {stat }}\left(T / 2, \vec{\theta}_{\ell}\right)} .
\end{gathered}
$$

For $\Phi_{8}^{\mathrm{HQET}}\left(\vec{\theta}_{1}, \vec{\theta}_{2}\right)$ in eq. (3.22):

$$
\begin{aligned}
& \tilde{\eta}_{8}\left(\vec{\theta}_{\ell}\right) \equiv \ln k_{\mathrm{A}_{2}}^{1, \text { stat }}\left(T / 2, \vec{\theta}_{\ell}\right), \\
& \tilde{\varphi}_{8}^{2}\left(\vec{\theta}_{\ell}, \vec{\theta}_{\mathrm{h}}\right) \equiv \frac{k_{\mathrm{A}_{2}}^{1, \text { kin }}\left(T / 2, \vec{\theta}_{\ell}, \vec{\theta}_{\mathrm{h}}\right)}{k_{\mathrm{A}_{2}}^{1, \text { stat }}\left(T / 2, \vec{\theta}_{\ell}\right)}, \\
& \tilde{\varphi}_{8}^{3}\left(\vec{\theta}_{\ell}\right) \equiv \frac{k_{\mathrm{A}_{2}}^{1, \text { sin }}\left(T / 2, \vec{\theta}_{\ell}\right)}{k_{\mathrm{A}_{2}}^{1, \text { stat }}\left(T / 2, \vec{\theta}_{\ell}\right)}, \\
& \tilde{\varphi}_{8}^{7}\left(\vec{\theta}_{\ell}, \vec{\theta}_{\mathrm{h}}\right) \equiv \frac{k_{\mathrm{A}_{2,1}}^{1}\left(T / 2, \vec{\theta}_{\ell}, \vec{\theta}_{\mathrm{h}}\right)}{k_{\mathrm{A}_{2}}^{1, \text { stat }}\left(T / 2, \vec{\theta}_{\ell}\right)}, \\
& \tilde{\varphi}_{8}^{8}\left(\vec{\theta}_{\ell}, \vec{\theta}_{\mathrm{h}}\right) \equiv \frac{k_{\mathrm{A}_{2,2}}^{1}\left(T / 2, \vec{\theta}_{\ell}, \vec{\theta}_{\mathrm{h}}\right)}{k_{\mathrm{A}_{2}}^{1, \text { stat }}\left(T / 2, \vec{\theta}_{\ell}\right)} .
\end{aligned}
$$

Note that the contribution from $\tilde{\varphi}_{8}^{8}\left(\vec{\theta}_{\ell}, \vec{\theta}_{\mathrm{h}}\right)$ vanishes at tree-level, because of its Dirac structure, for any choice of $\vec{\theta}_{\mathrm{h}}, \vec{\theta}_{\ell}$.

For $\Phi_{9}^{\mathrm{HQET}}\left(\vec{\theta}_{1}, \vec{\theta}_{2}, \vec{\theta}_{3}\right)$ in eq. $(3.23)$ :

$$
\begin{aligned}
& \tilde{\varphi}_{9}^{2}\left(\vec{\theta}_{\ell}, \vec{\theta}_{\mathrm{h}}\right) \equiv \tilde{\varphi}_{7}^{2}\left(\vec{\theta}_{\ell}, \vec{\theta}_{\mathrm{h}}\right), \\
& \tilde{\varphi}_{9}^{7}\left(\vec{\theta}_{\ell}, \vec{\theta}_{\mathrm{h}}\right) \equiv \tilde{\varphi}_{7}^{7}\left(\vec{\theta}_{\ell}, \vec{\theta}_{\mathrm{h}}\right), \\
& \tilde{\varphi}_{9}^{8}\left(\vec{\theta}_{\ell}, \vec{\theta}_{\mathrm{h}}\right) \equiv \tilde{\varphi}_{7}^{8}\left(\vec{\theta}_{\ell}, \vec{\theta}_{\mathrm{h}}\right), \\
& \tilde{\varphi}_{9}^{9}\left(\vec{\theta}_{\ell}, \vec{\theta}_{\mathrm{h}}\right) \equiv \sum_{k} \frac{f_{\mathrm{A}_{k, 3}}\left(T / 2, \vec{\theta}_{\ell}, \vec{\theta}_{\mathrm{h}}\right)}{f_{\overrightarrow{\mathrm{A}}}^{\text {stat }}\left(T / 2, \vec{\theta}_{\ell}\right)}, \\
& \tilde{\varphi}_{9}^{10}\left(\vec{\theta}_{\ell}, \vec{\theta}_{\mathrm{h}}\right) \equiv \sum_{k} \frac{f_{\mathrm{A}_{k, 4}}\left(T / 2, \vec{\theta}_{\ell}, \vec{\theta}_{\mathrm{h}}\right)}{f_{\overrightarrow{\mathrm{A}}}^{\text {stat }}\left(T / 2, \vec{\theta}_{\ell}\right)} .
\end{aligned}
$$

For $\Phi_{10}^{\mathrm{HQET}}\left(\vec{\theta}_{1}, \vec{\theta}_{2}, \vec{\theta}_{3}\right)$ in eq. (3.24):

$$
\tilde{\varphi}_{10}^{2}\left(\vec{\theta}_{\ell}, \vec{\theta}_{\mathrm{h}}\right) \equiv \tilde{\varphi}_{8}^{2}\left(\vec{\theta}_{\ell}, \vec{\theta}_{\mathrm{h}}\right),
$$




$$
\begin{aligned}
& \tilde{\varphi}_{10}^{7}\left(\vec{\theta}_{\ell}, \vec{\theta}_{\mathrm{h}}\right) \equiv \tilde{\varphi}_{8}^{7}\left(\vec{\theta}_{\ell}, \vec{\theta}_{\mathrm{h}}\right), \\
& \tilde{\varphi}_{10}^{8}\left(\vec{\theta}_{\ell}, \vec{\theta}_{\mathrm{h}}\right) \equiv \tilde{\varphi}_{8}^{8}\left(\vec{\theta}_{\ell}, \vec{\theta}_{\mathrm{h}}\right), \\
& \tilde{\varphi}_{10}^{9}\left(\vec{\theta}_{\ell}, \vec{\theta}_{\mathrm{h}}\right) \equiv \frac{k_{\mathrm{A}_{2,3}}^{1}\left(T / 2, \vec{\theta}_{\ell}, \vec{\theta}_{\mathrm{h}}\right)}{k_{\mathrm{A}_{2}}^{1, \mathrm{stat}}\left(T / 2, \vec{\theta}_{\ell}\right)}, \\
& \tilde{\varphi}_{10}^{10}\left(\vec{\theta}_{\ell}, \vec{\theta}_{\mathrm{h}}\right) \equiv \frac{k_{\mathrm{A}_{2,4}}^{1}\left(T / 2, \vec{\theta}_{\ell}, \vec{\theta}_{\mathrm{h}}\right)}{k_{\mathrm{A}_{2}}^{1, \text { stat }}\left(T / 2, \vec{\theta}_{\ell}\right)} .
\end{aligned}
$$

Again, the contribution from $\tilde{\varphi}_{10}^{8}\left(\vec{\theta}_{\ell}, \vec{\theta}_{\mathrm{h}}\right)$ and in addition from $\tilde{\varphi}_{10}^{10}\left(\vec{\theta}_{\ell}, \vec{\theta}_{\mathrm{h}}\right)$ vanish at treelevel.

For $\Phi_{11}^{\mathrm{HQET}}\left(\vec{\theta}_{1}\right)$ in eq. $(3.25)$ :

$$
\begin{aligned}
& \tilde{\eta}_{11}\left(\vec{\theta}_{\ell}\right) \equiv \ln \left(\frac{J_{\mathrm{A}_{1}}^{1 \text { stat }}\left(T / 2, \vec{\theta}_{\ell}, \vec{\theta}_{\ell^{\prime}}\right)}{\sqrt{K_{1}^{\ell \ell}\left(\vec{\theta}_{\ell}, \vec{\theta}_{\ell^{\prime}}\right) \times F_{1}^{\text {stat }}\left(\vec{\theta}_{\ell}\right)}}\right) \\
& \tilde{\varphi}_{11}^{2}\left(\vec{\theta}_{\ell}, \vec{\theta}_{\mathrm{h}}\right) \equiv\left(\frac{J_{\mathrm{A}_{1}}^{1 \operatorname{kin}\left(T / 2, \vec{\theta}_{\ell}, \vec{\theta}_{\ell^{\prime}}, \vec{\theta}_{\mathrm{h}}\right)}}{J_{\mathrm{A}_{1}}^{1 \text { stat }}\left(T / 2, \vec{\theta}_{\ell}, \vec{\theta}_{\ell^{\prime}}\right)}-\frac{1}{2} \frac{F_{1}^{\mathrm{kin}}\left(\vec{\theta}_{\ell}, \vec{\theta}_{\mathrm{h}}\right)}{F_{1}^{\mathrm{stat}}\left(\vec{\theta}_{\ell}\right)}\right) \\
& \tilde{\varphi}_{11}^{3}\left(\vec{\theta}_{\ell}\right) \equiv\left(\frac{J_{\mathrm{A}_{1}}^{1 \text { spin }}\left(T / 2, \vec{\theta}_{\ell}, \vec{\theta}_{\ell^{\prime}}\right)}{J_{\mathrm{A}_{1}}^{1 \text { stat }}\left(T / 2, \vec{\theta}_{\ell}, \vec{\theta}_{\ell^{\prime}}\right)}-\frac{1}{2} \frac{F_{1}^{\mathrm{sin}}\left(\vec{\theta}_{\ell}\right)}{F_{1}^{\mathrm{stat}}\left(\vec{\theta}_{\ell}\right)}\right) \\
& \tilde{\varphi}_{11}^{7}\left(\vec{\theta}_{\ell}, \vec{\theta}_{\mathrm{h}}\right) \equiv \frac{J_{\mathrm{A}_{1}, 1}^{1}\left(T / 2, \vec{\theta}_{\ell}, \vec{\theta}_{\ell^{\prime}}, \vec{\theta}_{\mathrm{h}}\right)}{J_{\mathrm{A}_{1}}^{1 \text { stat }}\left(T / 2, \vec{\theta}_{\ell}, \vec{\theta}_{\ell^{\prime}}\right)}, \\
& \tilde{\varphi}_{11}^{8}\left(\vec{\theta}_{\ell}, \vec{\theta}_{\mathrm{h}}\right) \equiv \frac{J_{\mathrm{A}_{1}, 2}^{1}\left(T / 2, \vec{\theta}_{\ell}, \vec{\theta}_{\ell^{\prime}}, \vec{\theta}_{\mathrm{h}}\right)}{J_{\mathrm{A}_{1}}^{1 \text { stat }}\left(T / 2, \vec{\theta}_{\ell}, \vec{\theta}_{\ell^{\prime}}\right)}
\end{aligned}
$$

The terms in the alternative observable $\Phi_{11}^{\prime \mathrm{HQET}}\left(\vec{\theta}_{1}\right)$ in eq. (3.26) explicitly read

$$
\begin{aligned}
& \tilde{\eta}_{11}^{\prime}\left(\vec{\theta}_{\ell}\right) \equiv \ln \left(\frac{f_{\overrightarrow{\mathrm{A}}}^{\text {stat }}\left(T / 2, \vec{\theta}_{\ell}\right)}{\left.\sqrt{F_{1}^{\text {stat }}\left(\vec{\theta}_{\ell}\right.}\right)}\right), \\
& \tilde{\varphi}_{11}^{\prime 2}\left(\vec{\theta}_{\ell}, \vec{\theta}_{\mathrm{h}}\right) \equiv \frac{f_{\overrightarrow{\mathrm{A}}}^{\text {kin }}\left(T / 2, \vec{\theta}_{\ell}, \vec{\theta}_{\mathrm{h}}\right)}{f_{\overrightarrow{\mathrm{A}}}^{\text {stat }}\left(T / 2, \vec{\theta}_{\ell}\right)}-\frac{1}{2} \frac{F_{1}^{\mathrm{kin}}\left(\vec{\theta}_{\ell}, \vec{\theta}_{\mathrm{h}}\right)}{F_{1}^{\text {stat }}\left(\vec{\theta}_{\ell}\right)}, \\
& \tilde{\varphi}_{11}^{\prime 3}\left(\vec{\theta}_{\ell}\right) \equiv \frac{f_{\overrightarrow{\mathrm{A}}}^{\mathrm{spin}}\left(T / 2, \vec{\theta}_{\ell}\right)}{f_{\overrightarrow{\mathrm{A}}}^{\mathrm{stat}}\left(T / 2, \vec{\theta}_{\ell}\right)}-\frac{1}{2} \frac{F_{1}^{\mathrm{spin}}\left(\vec{\theta}_{\ell}\right)}{F_{1}^{\mathrm{stat}}\left(\vec{\theta}_{\ell}\right)}, \\
& \tilde{\varphi}_{11}^{\prime 7}\left(\vec{\theta}_{\ell}, \vec{\theta}_{\mathrm{h}}\right) \equiv \tilde{\varphi}_{7}^{7}\left(\vec{\theta}_{\ell}, \vec{\theta}_{\mathrm{h}}\right), \\
& \tilde{\varphi}_{11}^{\prime 8}\left(\vec{\theta}_{\ell}, \vec{\theta}_{\mathrm{h}}\right) \equiv \tilde{\varphi}_{7}^{8}\left(\vec{\theta}_{\ell}, \vec{\theta}_{\mathrm{h}}\right) .
\end{aligned}
$$




\section{B Correlation functions and observables for the matching of the vector current}

We introduce the following boundary-to-boundary, boundary-to-bulk and three-point correlation functions

$$
\begin{aligned}
F_{1}^{\ell \ell}\left(\vec{\theta}_{\ell}, \vec{\theta}_{\ell^{\prime}}\right) & =-\frac{a^{12}}{2 L^{6}} \sum_{\mathbf{u}, \mathbf{v}, \mathbf{y}, \mathbf{z}}\left\langle\bar{\zeta}_{\ell}^{\prime}(\mathbf{u}) \gamma_{5} \zeta_{\ell^{\prime}}^{\prime}(\mathbf{v}) \bar{\zeta}_{\ell^{\prime}}(\mathbf{y}) \gamma_{5} \zeta_{\ell}(\mathbf{z})\right\rangle, \\
k_{\mathrm{V}_{0}}\left(x_{0}, \theta_{\ell}, \vec{\theta}_{\mathrm{h}}\right) & =i \frac{a^{6}}{6} \sum_{k} \sum_{\mathbf{y}, \mathbf{z}}\left\langle\left(V_{\mathrm{I}}\right)_{0}(x) \bar{\zeta}_{\mathrm{b}}(\mathbf{y}) \gamma_{k} \zeta_{\ell}(\mathbf{z})\right\rangle, \\
k_{\overrightarrow{\mathrm{V}}}\left(x_{0}, \theta_{\ell}, \vec{\theta}_{\mathrm{h}}\right) & =-\frac{a^{6}}{6} \sum_{k} \sum_{\mathbf{y}, \mathbf{z}}\left\langle\left(V_{\mathrm{I}}\right)_{k}(x) \bar{\zeta}_{\mathrm{b}}(\mathbf{y}) \gamma_{k} \zeta_{\ell}(\mathbf{z})\right\rangle, \\
k_{\mathrm{V}_{1}}^{1}\left(x_{0}, \theta_{\ell}, \vec{\theta}_{\mathrm{h}}\right) & =-\frac{a^{6}}{2} \sum_{\mathbf{y}, \mathbf{z}}\left\langle\left(V_{\mathrm{I}}\right)_{1}(x) \bar{\zeta}_{\mathrm{b}}(\mathbf{y}) \gamma_{1} \zeta_{\ell}(\mathbf{z})\right\rangle, \\
F_{\mathrm{V}_{0}}\left(x_{0}, \vec{\theta}_{\ell}, \vec{\theta}_{\ell^{\prime}}, \vec{\theta}_{\mathrm{h}}\right) & =-\frac{a^{15}}{2 L^{6}} \sum_{\mathbf{u}, \mathbf{v}, \mathbf{y}, \mathbf{z}, \mathbf{x}}\left\langle\bar{\zeta}_{\ell^{\prime}}^{\prime}(\mathbf{u}) \gamma_{5} \zeta_{\ell}^{\prime}(\mathbf{v})\left(V_{\mathrm{I}}\right)_{0}(x) \bar{\zeta}_{\mathrm{b}}(\mathbf{z}) \gamma_{5} \zeta_{\ell^{\prime}}(\mathbf{y})\right\rangle,
\end{aligned}
$$

where at least one component of the $\vec{\theta}$ angles in $k_{\mathrm{V}_{0}}$ should not be zero. With these correlators at hand we build the following matching observables

$$
\begin{array}{llr}
\Phi_{12}^{\mathrm{QCD}}\left(\vec{\theta}_{1}, \vec{\theta}_{2}\right) & \equiv \ln \left(\frac{k_{\mathrm{V}_{0}}\left(x_{0}, \vec{\theta}_{1}, \vec{\theta}_{1}\right)}{k_{\mathrm{V}_{0}}\left(x_{0}, \vec{\theta}_{2}, \vec{\theta}_{2}\right)}\right), & \left(T=L, x_{0}=T / 2\right), \\
\Phi_{13}^{\mathrm{QCD}}\left(\vec{\theta}_{1}, \vec{\theta}_{2}, \vec{\theta}_{3}\right) \equiv \ln \left(\frac{k_{\mathrm{V}_{0}}\left(x_{0}, \vec{\theta}_{1}, \vec{\theta}_{2}\right)}{k_{\mathrm{V}_{0}}\left(x_{0}, \vec{\theta}_{1}, \vec{\theta}_{3}\right)}\right), & \left(T=L, x_{0}=T / 2\right), \\
\Phi_{14}^{\mathrm{QCD}}\left(\vec{\theta}_{1}\right) \quad \equiv \ln \left(\frac{F_{\mathrm{V}_{0}}\left(x_{0}, \vec{\theta}_{1}, \vec{\theta}_{1}, \vec{\theta}_{1}\right)}{\sqrt{F_{1}\left(\vec{\theta}_{1}, \vec{\theta}_{1}\right) \times F_{1}^{\ell \ell}\left(\vec{\theta}_{1}, \vec{\theta}_{1}\right)}}\right),\left(T=L, x_{0}=T / 2\right), \\
\Phi_{15}^{\mathrm{QCD}}\left(\vec{\theta}_{1}, \vec{\theta}_{2}\right) & \equiv \ln \left(\frac{k_{\overrightarrow{\mathrm{V}}}\left(x_{0}, \vec{\theta}_{1}, \vec{\theta}_{1}\right)}{k_{\overrightarrow{\mathrm{V}}}\left(x_{0}, \vec{\theta}_{2}, \vec{\theta}_{2}\right)}\right), & \left(T=L, x_{0}=T / 2\right), \\
\Phi_{16}^{\mathrm{QCD}}\left(\vec{\theta}_{1}, \vec{\theta}_{2}\right) & \equiv \ln \left(\frac{k_{\mathrm{V}_{1}}^{1}\left(x_{0}, \vec{\theta}_{1}, \vec{\theta}_{1}\right)}{k_{\mathrm{V}_{1}}^{1}\left(x_{0}, \vec{\theta}_{2}, \vec{\theta}_{2}\right)}\right), & \left(T=L, x_{0}=T / 2\right), \\
\Phi_{17}^{\mathrm{QCD}}\left(\vec{\theta}_{1}, \vec{\theta}_{2}, \vec{\theta}_{3}\right) \equiv \ln \left(\frac{k_{\overrightarrow{\mathrm{V}}}\left(x_{0}, \vec{\theta}_{1}, \vec{\theta}_{2}\right)}{k_{\overrightarrow{\mathrm{V}}}\left(x_{0}, \vec{\theta}_{1}, \vec{\theta}_{3}\right)}\right), & \left(T=L, x_{0}=T / 2\right), \\
\Phi_{18}^{\mathrm{QCD}}\left(\vec{\theta}_{1}, \vec{\theta}_{2}, \vec{\theta}_{3}\right) \equiv \ln \left(\frac{k_{\mathrm{V}_{1}}^{1}\left(x_{0}, \vec{\theta}_{1}, \vec{\theta}_{2}\right)}{k_{\mathrm{V}_{1}}^{1}\left(x_{0}, \vec{\theta}_{1}, \vec{\theta}_{3}\right)}\right), & \left(T=L, x_{0}=T / 2\right),
\end{array}
$$




$$
\Phi_{19}^{\mathrm{QCD}}\left(\vec{\theta}_{1}\right) \equiv \ln \left(\frac{-k_{\overrightarrow{\mathrm{V}}}\left(x_{0}, \vec{\theta}_{1}, \vec{\theta}_{1}\right)}{\sqrt{K_{1}\left(\vec{\theta}_{1}, \vec{\theta}_{1}\right)}}\right),\left(T=L, x_{0}=T / 2\right) .
$$

An alternative for $\Phi_{14}^{\mathrm{QCD}}\left(\vec{\theta}_{1}\right)$, employing only two-point functions, is provided by

$$
\Phi_{14}^{\prime \mathrm{QCD}}\left(\vec{\theta}_{1}\right) \equiv \ln \left(\frac{k_{\mathrm{V}_{0}}\left(x_{0}, \vec{\theta}_{1}, \vec{\theta}_{1}\right)}{\sqrt{K_{1}\left(\vec{\theta}_{1}, \vec{\theta}_{1}\right)}}\right), \quad\left(T=L, x_{0}=T / 2\right) .
$$

In choosing the $\vec{\theta}$ angles for the results presented in this paper, we have made use of the fact that the correlators $k_{\mathrm{V}_{1,2}}^{1}$ and $k_{\mathrm{V}_{1,4}}^{1}$ vanish at tree-level, if the $x$-component of all the $\vec{\theta}$ 's are set to zero. This option helps in simplifying the system of equations and in making it entirely solvable by backward substitution.

The HQET expansion of the observables above can very easily be derived from the expansion of the quantities $\Phi_{4}^{\mathrm{QCD}}, \ldots, \Phi_{11}^{\mathrm{QCD}}$ in appendix A through the obvious replacements of correlators.

Open Access. This article is distributed under the terms of the Creative Commons Attribution License (CC-BY 4.0), which permits any use, distribution and reproduction in any medium, provided the original author(s) and source are credited.

\section{References}

[1] E. Lunghi and A. Soni, Possible evidence for the breakdown of the CKM-paradigm of CP-violation, Phys. Lett. B 697 (2011) 323 [arXiv:1010.6069] [INSPIRE].

[2] A. Lenz, U. Nierste, J. Charles, S. Descotes-Genon, H. Lacker et al., Constraints on new physics in $B-\bar{B}$ mixing in the light of recent LHCb data, Phys. Rev. D 86 (2012) 033008 [arXiv: 1203.0238] [INSPIRE].

[3] C. Tarantino, Flavor Lattice QCD in the Precision Era, PoS(ICHEP2012) 023 [arXiv: 1210.0474$]$ [INSPIRE].

[4] J.L. Rosner and S. Stone, Leptonic decays of charged pseudoscalar mesons - 2012, arXiv:1201.2401 [INSPIRE].

[5] ALPHA collaboration, J. Heitger and R. Sommer, Nonperturbative heavy quark effective theory, JHEP 02 (2004) 022 [hep-lat/0310035] [INSPIRE].

[6] M. Della Morte, P. Fritzsch and J. Heitger, Non-perturbative renormalization of the static axial current in two-flavour QCD, JHEP 02 (2007) 079 [hep-lat/0611036] [INSPIRE].

[7] B. Blossier, M. della Morte, N. Garron and R. Sommer, HQET at order 1/m: I. Non-perturbative parameters in the quenched approximation, JHEP 06 (2010) 002 [arXiv: 1001.4783] [INSPIRE].

[8] ALPHA collaboration, B. Blossier et al., HQET at order 1/m: II. Spectroscopy in the quenched approximation, JHEP 05 (2010) 074 [arXiv: 1004.2661] [INSPIRE].

[9] ALPHA collaboration, B. Blossier et al., HQET at order 1/m: III. Decay constants in the quenched approximation, JHEP 12 (2010) 039 [arXiv:1006.5816] [INSPIRE]. 
[10] ALPHA collaboration, B. Blossier et al., Parameters of Heavy Quark Effective Theory from $N_{f}=2$ lattice QCD, JHEP 09 (2012) 132 [arXiv:1203.6516] [INSPIRE].

[11] F. Bernardoni, B. Blossier, J. Bulava, M. Della Morte, P. Fritzsch et al., B-physics from HQET in two-flavour lattice QCD, PoS(LATTICE2012) 273 [arXiv:1210.7932] [INSPIRE].

[12] F. Bernardoni, B. Blossier, J. Bulava, M. Della Morte, P. Fritzsch et al., B-physics with $N_{f}=2$ Wilson fermions, PoS(LATTICE2013) 381 [arXiv:1309.1074] [INSPIRE].

[13] F. Bahr, F. Bernardoni, A. Ramos, H. Simma, R. Sommer et al., $B \rightarrow \pi$ form factor with 2 flavours of $O(a)$ improved Wilson quarks, PoS (LATTICE2012) 110 [arXiv:1210.3478] [INSPIRE].

[14] ALPHA collaboration, F. Bahr et al., $\left|V_{u b}\right|$ determination in lattice $Q C D$, PoS (ICHEP2012) 424 [arXiv: 1211.6327] [INSPIRE].

[15] ALPHA collaboration, work in progress.

[16] M. Lüscher, R. Narayanan, P. Weisz and U. Wolff, The Schrödinger functional: A renormalizable probe for non-Abelian gauge theories, Nucl. Phys. B 384 (1992) 168 [hep-lat/9207009] [INSPIRE].

[17] S. Sint, On the Schrödinger functional in QCD, Nucl. Phys. B 421 (1994) 135 [hep-lat/9312079] [INSPIRE].

[18] S. Sint, One loop renormalization of the QCD Schrödinger functional, Nucl. Phys. B 451 (1995) 416 [hep-lat/9504005] [INSPIRE].

[19] ALPHA collaboration, M. Kurth and R. Sommer, Renormalization and O(a) improvement of the static axial current, Nucl. Phys. B 597 (2001) 488 [hep-lat/0007002] [INSPIRE].

[20] D. Hesse and R. Sommer, A one-loop study of matching conditions for static-light flavor currents, JHEP 02 (2013) 115 [arXiv:1211.0866] [INSPIRE].

[21] L. Maiani, G. Martinelli and C.T. Sachrajda, Nonperturbative subtractions in the heavy quark effective field theory, Nucl. Phys. B 368 (1992) 281 [InSPIRE].

[22] R. Sommer, Introduction to Non-perturbative Heavy Quark Effective Theory, arXiv: 1008.0710 [INSPIRE].

[23] C.T. Sachrajda and G. Villadoro, Twisted boundary conditions in lattice simulations, Phys. Lett. B 609 (2005) 73 [hep-lat/0411033] [INSPIRE].

[24] M. Lüscher, S. Sint, R. Sommer and P. Weisz, Chiral symmetry and $O(a)$ improvement in lattice QCD, Nucl. Phys. B 478 (1996) 365 [hep-lat/9605038] [INSPIRE].

[25] S. Sint and P. Weisz, Further results on $O($ a) improved lattice QCD to one loop order of perturbation theory, Nucl. Phys. B 502 (1997) 251 [hep-lat/9704001] [INSPIRE].

[26] ALPHA collaboration, D. Guazzini, H.B. Meyer and R. Sommer, Non-perturbative renormalization of the chromo-magnetic operator in Heavy Quark Effective Theory and the $B^{*}-B$ mass splitting, JHEP 10 (2007) 081 [arXiv:0705.1809] [INSPIRE].

[27] E. Eichten and B.R. Hill, An Effective Field Theory for the Calculation of Matrix Elements Involving Heavy Quarks, Phys. Lett. B 234 (1990) 511 [INSPIRE].

[28] M. Della Morte, A. Shindler and R. Sommer, On lattice actions for static quarks, JHEP 08 (2005) 051 [hep-lat/0506008] [INSPIRE]. 
[29] M. Lüscher and P. Weisz, $O(a)$ improvement of the axial current in lattice $Q C D$ to one loop order of perturbation theory, Nucl. Phys. B 479 (1996) 429 [hep-lat/9606016] [INSPIRE].

[30] ALPHA collaboration, P. Korcyl, in preparation.

[31] ALPHA collaboration, P. Korcyl, Fixing the parameters of Lattice HQET including $1 / m_{b}$ terms, PoS (Beauty2013) 071 [arXiv: 1307.5080] [INSPIRE]. 